\title{
On the Operationalization of Graph Queries with Generalized Discrimination Networks
}

Thomas Beyhl, Dominique Blouin, Holger Giese, Leen Lambers

Technische Berichte Nr. 106

des Hasso-Plattner-Instituts für

Softwaresystemtechnik

an der Universität Potsdam

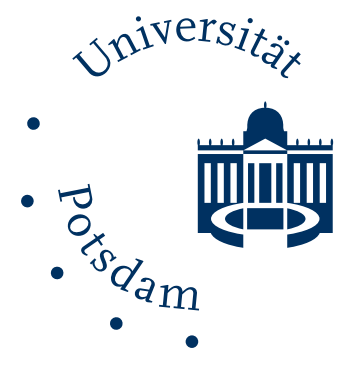



Technische Berichte des Hasso-Plattner-Instituts für Softwaresystemtechnik an der Universität Potsdam 

Technische Berichte des Hasso-Plattner-Instituts für Softwaresystemtechnik an der Universität Potsdam | 106

Thomas Beyhl | Dominique Blouin | Holger Giese I Leen Lambers

\section{On the Operationalization of Graph Queries with Generalized Discrimination Networks}


Bibliografische Information der Deutschen Nationalbibliothek

Die Deutsche Nationalbibliothek verzeichnet diese Publikation in der Deutschen Nationalbibliografie; detaillierte bibliografische Daten sind im Internet über http://dnb.dnb.de/ abrufbar.

Universitätsverlag Potsdam 2016

http://verlag.ub.uni-potsdam.de/

Am Neuen Palais 10, 14469 Potsdam

Tel.: +49 (0)331977 2533 / Fax: 2292

E-Mail: verlag@uni-potsdam.de

Die Schriftenreihe Technische Berichte des Hasso-Plattner-Instituts für Softwaresystemtechnik an der Universität Potsdam wird herausgegeben von den Professoren des Hasso-Plattner-Instituts für Softwaresystemtechnik an der Universität Potsdam.

ISSN (print) 1613-5652

ISSN (online) 2191-1665

Das Manuskript ist urheberrechtlich geschützt.

Druck: docupoint GmbH Magdeburg

\section{ISBN 978-3-86956-372-5}

Zugleich online veröffentlicht auf dem Publikationsserver der Universität Potsdam: URN urn:nbn:de:kobv:517-opus4-96279

http:/ /nbn-resolving.de/urn:nbn:de:kobv:517-opus4-96279 
Graph queries have lately gained increased interest due to application areas such as social networks, biological networks, or model queries. For the relational database case the relational algebra and generalized discrimination networks have been studied to find appropriate decompositions into subqueries and ordering of these subqueries for query evaluation or incremental updates of query results. For graph database queries however there is no formal underpinning yet that allows us to find such suitable operationalizations. Consequently, we suggest a simple operational concept for the decomposition of arbitrary complex queries into simpler subqueries and the ordering of these subqueries in form of generalized discrimination networks for graph queries inspired by the relational case. The approach employs graph transformation rules for the nodes of the network and thus we can employ the underlying theory. We further show that the proposed generalized discrimination networks have the same expressive power as nested graph conditions. 



\section{Contents}

\begin{tabular}{lr}
\hline 1. Introduction & 8
\end{tabular}

\begin{tabular}{lr}
\hline 2. Prerequisites & 10
\end{tabular}

$\begin{array}{ll}\text { 3. Generalized Discrimination Networks } & 15\end{array}$

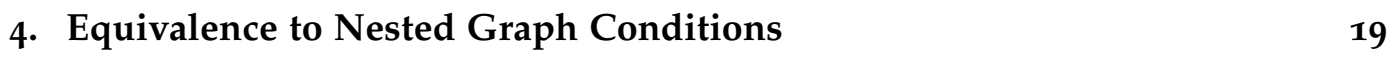

\begin{tabular}{ll}
\hline 5. Discussion & 23
\end{tabular}

6. Conclusion and Future Work 24

\begin{tabular}{|ll}
\hline A. Omitted Results and Proofs & 28
\end{tabular}

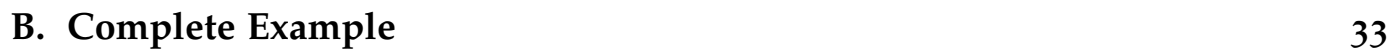




\section{Introduction}

The model of typed graphs and related graph queries to explore existing graphs and their properties has lately gained increased importance due to application areas of increasing relevance such as social networks, biological networks, and model queries [15] and technologies like graph databases [2] or model-driven development [4] where graphs rather than relations are the main characteristics of the employed models and queries.

While the definition of typed graphs by means of schemas, metamodels, or grammars is a formally well studied topic, there is yet no clear formal underpinning for graph queries concerning their specification as well as their operationalization (cf. [2, 18]). For the operationalization of the query evaluation and incremental query updates of relational queries the relational calculus [1] and generalized discrimination networks (GDN) have been suggested (cf. [14]) as a formal framework to study which decomposition into subqueries and ordering of these subqueries is most appropriate. As depicted in Figure I (a), in such a network each network node (numbered block) is responsible for evaluating a subquery and for this purpose it may compose subquery evaluations of nodes it depends on. The overall result is then the query evaluation of the terminal node. However, such a formal framework does not exist for graph queries so far.
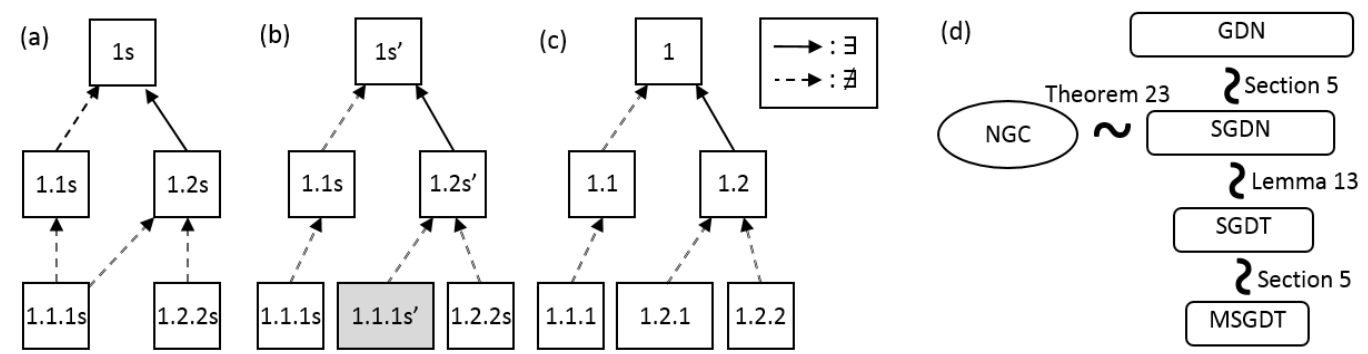

Figure 1: GDNs in form of a SGDN (a) and SGDTs (b)(c) for a social network query

Consequently, inspired by the relational case we suggest motivated by our practical work on view maintenance for graph databases [6] a simple operational concept for the decomposition of arbitrary complex graph queries into a suitable ordering of simpler subqueries in form of GDNs. Rather than considering one particular kind of GDN with particular network nodes, we suggest employing graph transformation 
(GT) rules for these network nodes such that we are also able to employ the well understood GT theory [9] as a basis. The basic idea to define our notion of GDN related to GT systems is to employ extra marking nodes and edges to encode the results of subqueries and specific graph transformation rules to describe the propagation behavior of the network nodes via creating and reading markings.

We study in this paper what are the core ingredients required to approach graph query evaluation based on an operational specification using the above-described GDNs while having the same expressiveness as declarative graph queries based on nested graph conditions (NGC) [13]. The latter have the expressive power of first order logic on graphs and constitute as such a natural formal foundation for patternbased graph queries.

We assume in the following that a graph query is characterized by a request graph $L$ delivering its answers in form of a set of matches for $L$ into the queried graph $G$ fulfilling some additional properties as described in the graph query ${ }^{1}$ Based on the answer set semantics we were able to establish equivalence of NGCs with GDNs including different specific subsets such as so-called simple GDNs (SGDNs), simple tree-like GDNs (SGDT), and minimal SGDTs (MSGDT). In particular as depicted in Fig. 1 (d), as a main result we established the equivalence between NGCs and SGDNs and in addition showed that all GDN variants are equally expressive.

The paper is structured as follows: We first introduce our running example as well as the foundations concerning typed graphs, graph queries in their generic form, NGCs, and GT in Section 2. Then, in Section 3 operational graph queries in form of GDNs are defined and it is shown how to transform SGDNs into trees (SGDTs). That SGDNs and declarative queries based on NGCs have the same expressive power follows in Section 4 and we discuss the different variants of GDNs concerning their expressiveness and applicability w. r.t. optimization and incremental updates for graph queries in Section 5 . Finally, we conclude the paper and provide an outlook on planned future work.

This report is an extended version of [5]. In addition, it includes an appendix with a complete description of our running example as well as omitted results and proofs.

\footnotetext{
${ }^{1}$ It is to be noted that a simple record as provided by an SQL-statement is also a special form of graph where no links are included. Moreover, while in practice the requested number of answers is often limited to a fixed upper bound of answers, for our more theoretical considerations in this paper, we can assume w. l.o.g. that all matches of $L$ for $G$ that fulfill the additional properties that must hold are building the correct set of answers.
} 


\section{Prerequisites}

After outlining our running example, we will introduce typed graphs, based on that a generic notion of graph query (language) together with the concept of equivalence, the notion of graph conditions with arbitrary nesting level (NGCs), and GT systems. Moreover, we introduce in particular the answer set of graph queries based on NGCs.

(a) knows

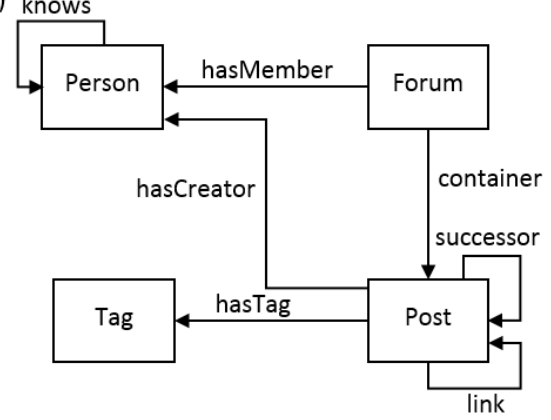

(b)

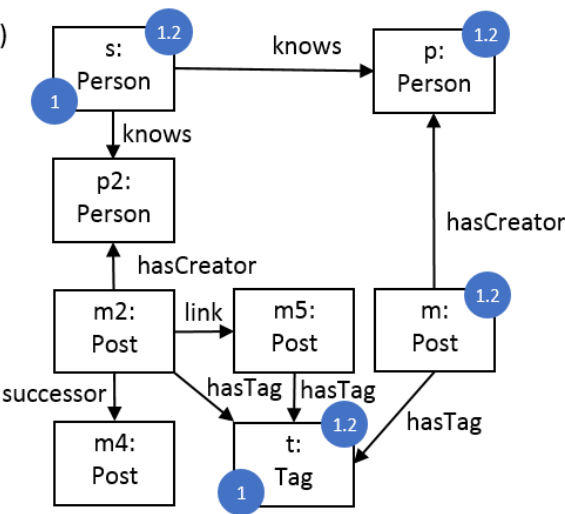

Figure 2: Excerpt of social network type graph and an example graph $G$

Example I (social network query). As running example we use a social network model and a slightly adjusted graph query employed by the LDBC benchmark 16. A class diagram outlining the possible graph models as well as an example graph to apply the query are depicted in Figure 2 (a) resp. (b). The considered complex graph query looks for pairs of Tags and Persons (1) such that the Tag is new in the Posts by a friend of this Person. To be a Post of a friend, the Post must be from a second Person the Person knows (1.2). In order to be new, the Tag must be linked in the latest Post of the second Person (and thus in a Post that has no successor Post) (1.2.2) and there has to be no former Post by any other or the same friend that is not her last one and where the same Tag has been already used (1.1). In both cases only Tags that are not simply inherited from a linked Post should be considered (1.1.1 and 1.2.1). Note that the employed numbering of the conditions relates to the tree-like network depicted in Figure 1 (c). Occurrences for the positive sentences (1) and (1.2) in the example graph are depicted accordingly as markers in form of blue circles with the respective number in Figure 2 (b). The circular blue markers (1) on the graph denote the occurrence of the request graph consisting of the person s and tag t. Marker (1.2) denotes the extra condition that the searched tag $t$ must be attached (hasTag) to a post created by person $p$ that is known 
by person s. Note that the markers (1) denote the only correct answer for the query. Thereby the required match for the positive subquery (1.2) depicted by the markers (1.2) is such that indeed no match exists for the negative subsubqueries (1.2.1) and (1.2.2). Furthermore, as required no match for the negative subquery (1.1) consistent with (1) exists such that no match for the negative subsubquery (1.1.1) of (1.1) can be found. Consequently, no match for (1.1) is visualized.

We briefly reintroduce the notion of typed graphs and graph morphisms [9]. A graph $G=\left(G^{V}, G^{E},{ }_{s}{ }, t^{G}\right)$ consists of a set $G^{V}$ of nodes, a set $G^{E}$ of edges, a source function $s^{G}: G^{E} \rightarrow G^{V}$, and a target function $t^{G}: G^{E} \rightarrow G^{V}$. Given the graphs $G=\left(G^{V}, G^{E}, s^{G}, t^{G}\right)$ and $H=\left(H^{V}, H^{E}, s^{H}, t^{H}\right)$, a graph morphism $f: G \rightarrow H$ is a pair of mappings, $f^{V}: G^{V} \rightarrow H^{V}, f^{E}: G^{E} \rightarrow H^{E}$ such that $f^{V} \circ s^{G}=s^{H} \circ f^{E}$ and $f^{V} \circ t^{G}=t^{H} \circ f^{E}$. A graph morphism $f: G \rightarrow H$ is a monomorphism if $f^{V}$ and $f^{E}$ are injective mappings. Finally, two graph morphisms $m: H \rightarrow G$ and $m^{\prime}: H^{\prime} \rightarrow G$ are jointly epimorphic if $m^{V}\left(H^{V}\right) \cup m^{\prime V}\left(H^{\prime V}\right)=G^{V}$ and $m^{E}\left(H^{E}\right) \cup m^{\prime E}\left(H^{\prime E}\right)=G^{E}$. A type graph is a distinguished graph $T G=\left(T G^{V}, T G^{E}, s^{T G}, t^{T G}\right) . T G^{V}$ and $T G^{E}$ are called the vertex and the edge type alphabets, respectively. A tuple $(G$, type $)$ of a graph $G$ together with a graph morphism type : $G \rightarrow T G$ is then called a typed graph. Given typed graphs $G_{1}^{T}=\left(G_{1}\right.$, type $\left._{1}\right)$ and $G_{2}^{T}=\left(G_{2}\right.$, type $)$, a typed graph morphism $f: G_{1}^{T} \rightarrow G_{2}^{T}$ is a graph morphism $f: G_{1} \rightarrow G_{2}$ such that type $e_{2} \circ f=$ type $_{1}$. We further denote the set of all graphs typed over some type graph $T G$ by $\mathcal{L}(T G)$.

An example for a typed graph $G$ and the type graph $T G$ related to the social network query Example 1 are depicted in Figure 2.

In the rest of the paper we will compare the answer sets of graph queries to analyze them for equivalence. Since we will compare queries stemming from different query languages, we introduce here a generic notion of query (language) equivalence that we will refine in the rest of the paper to particular queries and query languages. As the most generic form of a graph query language we just assume that it consists of a set of graph queries, where each graph query is characterized by a request graph $L$ typed over some type graph $T G$. The query then expresses some extra properties that need to hold for the request graph $L$ that is searched for in the queried graph $G$. The answer set for this query then describes all matches of $L$ in the queried graph that fulfill these extra properties.

Definition 1 (graph query (language)). Given a type graph $T G$, then a graph query is characterized by a so-called request graph $L$, which is a finite graph typed over TG. A graph query language is a set of graph queries.

Definition 2 (answer set mapping, equivalence). Given some graph query language $\mathcal{L}$, an answer set mapping ans for $\mathcal{L}$ maps each pair $\left(q_{L}, G\right)$ with $q_{L}$ a graph query in $\mathcal{L}$ with request graph $L$ typed over $T G$ and $G$ a graph from $\mathcal{L}(T G)$ to a set of graph 
morphisms typed over TG with domain L and co-domain G.

Given queries $q_{L}$ and $q_{L}^{\prime}$ for some request graph $L$ typed over $T G$ belonging to the graph query languages $\mathcal{L}$ and $\mathcal{L}^{\prime}$ with answer set mappings ans and ans', resp., then $q_{L}$ and $q_{L}^{\prime}$ are equivalent if for every graph $G$ in $\mathcal{L}(T G)$ it holds that ans $\left(q_{L}, G\right)=\operatorname{ans}^{\prime}\left(q_{L}^{\prime}, G\right)$. Two graph query languages $\mathcal{L}$ and $\mathcal{L}^{\prime}$ are equivalent if for any query $q_{L} \in \mathcal{L}$ for some request graph $L$ there exists some query $q_{L}^{\prime} \in \mathcal{L}^{\prime}$ for $L$ such that $q_{L} \sim q_{L}^{\prime}$ and vice versa. We denote equivalence also with $\sim$.

We reintroduce the notion of nested graph conditions (NGC) from [13], since they represent the declarative kind of graph queries that we will consider in this paper. Given a finite graph $L$, a nested graph condition (NGC) over $L$ is defined inductively as follows: (1) true is a NGC over L. We say that true has nesting level 0. (2) For every morphism $a: L \rightarrow L^{\prime}$ and NGC $c_{L^{\prime}}$ over a finite graph $L^{\prime}$ with nesting level $n$ such that $n \geq 0, \exists\left(a, c_{L^{\prime}}\right)$ is a NGC over $L$ with nesting level $n+1$. (3) Given NGCs over $L, c_{L}$ and $c_{L}^{\prime}$, with nesting level $n$ and $n^{\prime}$, respectively, $\neg c_{L}$ and $c_{L} \wedge c_{L}^{\prime}$ are NGCs over $L$ with nesting level $n$ and $\max \left(n, n^{\prime}\right)$, respectively. We restrict ourselves to finite NGCs, i.e. each conjunction of NGCs is finite. We define when a morphism $q: L \rightarrow G$ satisfies a NGC $c_{L}$ over $L$ inductively: (1) Every morphism $q$ satisfies true. (2) A morphism $q$ satisfies $\exists\left(a, c_{L^{\prime}}\right)$, denoted $q \models \exists\left(a, c_{L^{\prime}}\right)$, if there exists a monomorphism $q^{\prime}: L^{\prime} \rightarrow G$ such that $q^{\prime} \circ a=q$ and $q^{\prime}=c_{L^{\prime}}$. (3) A morphism $q$ satisfies $\neg c_{L}$ if it does not satisfy $c_{L}$ and satisfies $\wedge_{i \in I} c_{L, i}$ if it satisfies each $c_{L, i}$ $(i \in I)$. Note that false, $\vee$, and $\Rightarrow$ can be mapped as usual to the introduced logical connectives. Moreover we abbreviate $\exists\left(\varnothing \rightarrow L^{\prime}, c_{L^{\prime}}\right)$ with $\exists\left(L^{\prime}, c_{L^{\prime}}\right), \exists(a$, true $)$ with $\exists a$ and $\forall\left(a, c_{L^{\prime}}\right)$ with $\neg \exists\left(a, \neg c_{L^{\prime}}\right)$. NGCs can be equipped with typing over a given type graph $T G$ as usual [9] by adding typing morphisms from each graph to $T G$ and by requiring type-compatibility with respect to $T G$ for each graph morphism $?^{2}$

Definition $3\left(\mathcal{L}_{N G C}, a n s_{N G C}\right)$. The graph query language $\mathcal{L}_{N G C}$ is the set of all NGCs. Given some NGC $c_{L}$ over $L, L$ represents the so-called request graph. The answer set mapping ans ${ }_{N G C}$ for $\mathcal{L}_{N G C}$ is given by

$$
\operatorname{ans}_{N G C}\left(c_{L}, G\right)=\left\{q: L \rightarrow G \mid q \text { is a monomorphism and } q \mid=c_{L}\right\}
$$

with $c_{L} \in \mathcal{L}_{N G C}$ a NGC with $L$ typed over some type graph $T G$ and $G$ in $\mathcal{L}(T G)$.

An example NGC for the social network query of Example 1 , where the subconditions refer to the introduced numbering, is the following: $c_{1}=c_{1.1} \wedge c_{1.2}$

\footnotetext{
${ }^{2}$ W.l.o.g. we restrict our notion of condition satisfaction to the existence of monomorphisms. In particular, in [13] it is shown how to translate conditions relying on general morphism matching/satisfaction into equivalent conditions relying on monomorphism matching/satisfaction and the other way round.
} 

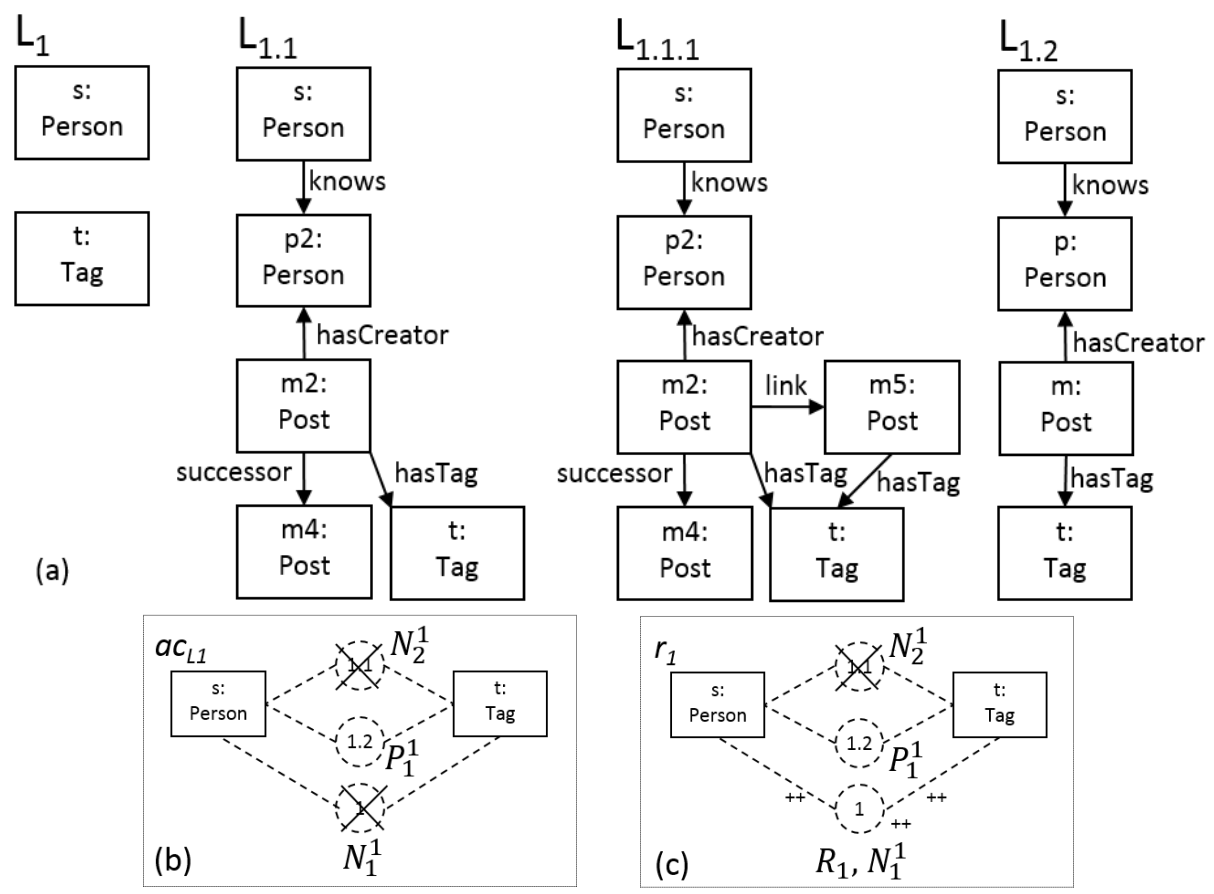

Figure 3: Graphs for the NGC $c_{1}$ and its subconditions (a) and the application condition $a c_{L_{1}}=\exists\left(L_{1} \rightarrow P_{1}^{1}\right) \wedge \nexists\left(L_{1} \rightarrow N_{1}^{1}\right) \wedge \nexists\left(L_{1} \rightarrow N_{2}^{1}\right)$ (b) and simple marking rule $r_{1}=\left(L_{1} \rightarrow R_{1}, a c_{L_{1}}\right)$ (c) 
with $c_{1.1}=\neg \exists\left(n_{1.1}: L_{1} \rightarrow L_{1.1}, c_{1.1 .1}\right), c_{1.2}=\exists\left(p_{1.2}: L_{1} \rightarrow L_{1.2}, c_{1.2 .1} \wedge c_{1.2 .2}\right)$, $c_{1.1 .1}=\neg \exists\left(n_{1.1 .1}: L_{1.1} \rightarrow L_{1.1 .1}\right.$, true $), c_{1.2 .1}=\neg \exists\left(n_{1.2 .1}: L_{1.2} \rightarrow L_{1.2 .1}\right.$, true $)$, and $c_{1.2 .2}=\neg \exists\left(n_{1.2 .2}: L_{1.2} \rightarrow L_{1.2 .2}\right.$, true $)$. The graphs $L_{1}, L_{1.1}, L_{1.1 .1}$, and $L_{1.2}$ are depicted exemplarily (see Section B for the complete example) in Figure 3 (a). Morphisms are implied by equally named objects.

As foundation for an operational graph query evaluation we will employ typed GT systems with priorities. We start with reintroducing GT and thereby assume the double-pushout approach (DPO) with injective matching and non-deleting rules [9] with application conditions of arbitrary nesting level (AC) [13]. A plain GT rule $p: L \rightarrow R$ is a graph monomorphism. We say that the graphs $L$ and $R$ are the left-hand side (LHS) and right-hand side (RHS) of the rule, respectively. A GT rule $r=\left\langle p\right.$, ac $\left._{L}\right\rangle$ consists of a plain rule $p: L \rightarrow R$ and a so-called application condition $\mathrm{ac}_{L}$ being a graph condition over $L$. If the application condition $\mathrm{ac}_{L}=$ $\wedge_{i \in I} \exists p_{i} \wedge \wedge_{j \in J} \nexists n_{j}$, then we say that $\exists p_{i}$ or $\neg \exists n_{j}$ is a positive application condition (PACs) or negative application condition (NAC) over $L$, respectively. A rule $r$ is applicable to a graph $G$ via a graph monomorphism $m: L \rightarrow G$ if $m \models \mathrm{ac}_{L}$. A direct GT via rule $r=\left\langle p, \mathrm{ac}_{L}\right\rangle$ consists of a pushout over $p$ and $m$ such that $m \models \mathrm{ac}_{L}$. If there exists a direct transformation from $G$ to $G^{\prime}$ via rule $r$ and match $m$, we write $G \Rightarrow_{m, r} G^{\prime}$. If we are only interested in the rule $r$, we write $G \Rightarrow_{r} G^{\prime}$. If a rule $r$ in a set of rules $\mathcal{R}$ exists such that there exists a direct transformation via rule $r$ from $G$ to $G^{\prime}$, we write $G \Rightarrow_{\mathcal{R}} G^{\prime}$. A $G T$, denoted as $G_{0} \Rightarrow^{*} G_{n}$, is a sequence $G_{0} \Rightarrow G_{1} \Rightarrow \cdots \Rightarrow G_{n}$ of $n \geq 0$ direct GT. GT rules and GTs can be equipped with typing over a given type graph TG as usual [9] by adding typing morphisms from each graph to TG and by requiring type-compatibility with respect to TG for each graph morphism.

An example for a GT rule with AC in the context of the social network query of Example $\mathrm{I}$ is $r_{1}=\left(L_{1} \rightarrow R_{1}, a c_{L_{1}}\right)$ as depicted in Figure 3 (c) following the compact notation where all graphs are embedded into a single one. In particular, $a c_{L_{1}}=\exists\left(L_{1} \rightarrow P_{1}^{1}\right) \wedge \nexists\left(L_{1} \rightarrow N_{1}^{1}\right) \wedge \nexists\left(L_{1} \rightarrow N_{2}^{1}\right)$ is depicted more precisely in Figure 3 (b). ++ denotes elements that are created by the rule, the additional (dashed) elements forbidden by a NAC are crossed out and the extra elements required by a PAC are dashed as well. These crosses for NAC $N_{1}^{1}$ are omitted from the rule visualization in Figure 3 (c) as it equals $R_{1}$. Note that we use in this example in addition to the node types defined in the type graph depicted in Figure 2 (a) (solid rectangles) already some additional marking node (dashed circles) and edge types (dashed lines) that will be introduced later.

A graph transformation system (GTS) gts $=(\mathcal{R}, \mathrm{TG})$ consists of a set of rules $\mathcal{R}$ typed over a type graph TG. If a rule $r$ in $\mathcal{R}$ of gts exists such that a direct transformation $G \Rightarrow_{r} G^{\prime}$ via $r$ exists, we also write $G \Rightarrow_{\mathrm{gts}} G^{\prime}$. If for some graph $G$ it holds that $r$ is not applicable to $G$, then we write $G \nRightarrow_{r}$. Moreover, if no rule 
in gts exists that is applicable to $G$, then we write $G \nRightarrow \nRightarrow_{\text {gts. }}$. A GTS with priorities gts $_{p}=((\mathcal{R}, T G), p)$ consists of a GTS $(\mathcal{R}, T G)$ and a transitive and asymmetric

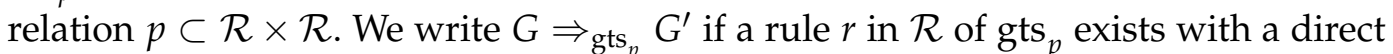
transformation $G \Rightarrow{ }_{r} G^{\prime}$ such that $\nexists r^{\prime} \in \mathcal{R}:\left(r, r^{\prime}\right) \in p \wedge G \Rightarrow_{r^{\prime}} G^{\prime \prime}$. For a GTS with priorities gts gnd $_{p}$ an initial graph $G_{0}$ the set of reachable graphs $\operatorname{REACH}\left(\mathrm{gts}_{p}, G_{0}\right)$ is defined as $\left\{G \mid G_{0} \Rightarrow_{\text {gts }_{p}}^{*} G\right\}$ and the set of terminal reachable graphs $\operatorname{TERM}\left(\right.$ gts $\left._{p}, G_{0}\right)$ is defined as $\left\{G \mid G \in \operatorname{REACH}\left(\mathrm{gts}_{p}, G_{0}\right): G \not \Rightarrow_{g t s_{p}}\right\}$.

\section{Generalized Discrimination Networks}

In the following we introduce our suggestion for the operationalization of graph queries employing generalized discrimination networks with network nodes based on GT rules.

Example 2 (GDN (informal)). A possible GDN for the social network query Example 1 is depicted in Figure 1 (a). Node 1.1.1s and 1.2.2s produce their output independently. Then, node $1.1 \mathrm{~s}$ and $1.2 \mathrm{~s}$ can compute the output depending on the output of these two other nodes. Finally, the terminal node 1 s can compute its output based on the output of the nodes $1.1 s$ and 1.2s. We further distinguish in Figure 1 (a) positive and negated dependencies accordingly visualized by arrows with a single solid line when representing a PAC $(\exists)$ and by arrows with a single dashed line when representing a NAC ( $\nexists$ ).

Our queried graph $G$ typed over TG will be marked with so-called marking nodes and edges to keep track of (sub-)query answer sets. In particular, so-called marking rules in a GDN will take care of that. A (simple) marking rule $r_{i}$ is a restricted form of GT rule typed over a marking type graph $T G^{\prime}$. The latter is equal to TG but for each marking rule $r_{i}$ it is extended with a so-called marking node type $t_{i}$ as well as an marking edge type $t_{v}$ per node $v$ present in $r_{i}{ }^{\prime}$ s LHS $L_{i}$. This allows $r_{i}$ to mark each node $v$ from $L_{i}$ by adding a marking node $i$ uniquely corresponding to $r_{i}$ via its marking node type $t_{i}$, called the defined type, and by adding a marking edge $e_{v}$ from this special marking node $i$ to each node $v$ in $L_{i}$. These marking edges encode again via their type $t_{v}$ which node $v$ in $L_{i}$ they mark. Finally the application conditions in each marking rule allow for referring to the marking elements (and therefore indirectly to already matched elements) created by other rules.

The required extension for the type graph $T G$ for the social network query Example 2 for rule $r_{1}$, which captures that a s:Person and t:Tag exist for which additional conditions must hold, are depicted in Figure 3 (c). Additional nodes visualized as circles with number $1,1.1$, and 1.2, where 1 denotes the created 
marking node of the rule $r_{1}$ and 1.1 and 1.2 are marking nodes of the other rules $r_{1.1}$ and $r_{1.2}$ all use types in $T G^{\prime}$ but not $T G$. The edges between the circles and the rectangles also belong to $T G^{\prime}$ but not $T G$. We do not visualize their direction, since they always point to nodes of a type from $T G$.

Definition 4 (marking type graph). Given a set of graphs $\left(L_{i}\right)_{i \in I}$ typed over TG via type $_{i}: L_{i} \rightarrow T G$, the marking type graph $T G^{\prime}$ for $\left(L_{i}\right)_{i \in I}$ has node set $T G^{\prime V}=$ $T G^{V} \uplus\left\{t_{i} \mid i \in I\right\}$ and edge set $T G^{\prime E}=T G^{E} \uplus\left\{t_{v} \mid v \in L_{i}^{V}, i \in I\right\}$ s.t. $s^{T G^{\prime}}(e)=s^{T G}(e)$ and $t^{T G^{\prime}}(e)=t^{T G}(e)$ for $e \in T G^{E}$ and $s^{T G^{\prime}}\left(t_{v}\right)=t_{i}$ and $t^{T G^{\prime}}\left(t_{v}\right)=\operatorname{type}_{i}^{V}(v)$ for each $v \in L_{i}^{V}$ and $i \in I$ otherwise. We say that the nodes in $\left\{t_{i} \mid i \in I\right\}$ are marking node types and edges in $\left\{t_{v} \mid v \in L_{i}^{V}, i \in I\right\}$ are marking edge types, respectively. Given a graph $G$ typed over $T G^{\prime}$, then we say that a node or edge in $G$ such that its type equals a marking node or edge type in $T G^{\prime}$ is a marking node or edge in $G$, resp..

Definition 5 ((simple) marking rule, defined type). Given a set of graphs $\left(L_{i}\right)_{i \in I}$ typed over TG via type $e_{i}: L_{i} \rightarrow T G$, a marking rule (MR) is a GT rule $r_{i}=\left\langle p_{i}: L_{i} \rightarrow\right.$ $\left.R_{i}, \nexists p_{i} \wedge c_{L_{i}}\right\rangle$ typed over the marking type graph $T G^{\prime}$ for $\left(L_{i}\right)_{i \in I}$ such that (1) $L_{i}$ inherits its typing from type $L_{L_{i}}$ (2) $R_{i}^{V}=L_{i}^{V} \uplus\{i\}$ with $i$ of type $t_{i}$ the so-called marking node and $t_{i}$ the so-called defined type of rule $r_{i}$, and (3) $R_{i}^{E}=L_{i}^{E} \uplus\left\{e_{v} \mid v \in L_{i}^{V}\right\}$ such that each $e_{v}$ has type $t_{v}$ and $s^{R_{i}}\left(e_{v}\right)=i$ and $t^{R_{i}}\left(e_{v}\right)=v$.

A simple marking rule $(S M R)$ is a marking rule where the application condition $c_{L_{i}}=\bigwedge_{j \in J}\left(\exists p_{j}: L_{i} \rightarrow P_{j}\right) \wedge \bigwedge_{k \in K}\left(\nexists n_{k}: L_{i} \rightarrow N_{k}\right)$ such that for each $j \in J$ and $k \in K$ it holds that $P_{j}^{V} \backslash\left(p_{j}\left(L_{i}\right)\right)^{V}$ and $N_{k}^{V} \backslash\left(n_{k}\left(L_{i}\right)\right)^{V}$, resp., consist of exactly one marking node.

In addition to the defined type of its created marking node each marking rule induces so-called referred types in the marking type graph. Based on these referred and defined types of MRs we define a dependency relation between MRs.

Definition 6 (referred types, dependency relation). Given a set of graphs $\left(L_{i}\right)_{i \in I}$ typed over TG and a (simple) marking rule $r_{i}=\left\langle p_{i}: L_{i} \rightarrow R_{i}, \nexists p_{i} \wedge c_{L_{i}}\right\rangle$ typed over the marking type graph $T G^{\prime}$ for $\left(L_{i}\right)_{i \in I}$ the set of referred types $r t\left(r_{i}\right)$ is the set of all node types in $T G^{\prime V}$ for nodes occurring in some (co-)domain graph of a morphism employed in $c_{L_{i}}$.

Given a GTS $\left(\mathcal{R}=\left(r_{i}\right)_{i \in I}, T G^{\prime}\right)$ with each rule $r_{i}$ a (simple) marking rule, a dependency relation $\rightsquigarrow_{d} \subseteq \mathcal{R} \times \mathcal{R}$ consists of all rule pairs $\left(r_{i}, r_{j}\right)$ such that the defined type $t_{j}$ of rule $r_{j}$ belongs to the set of referred types $r t\left(r_{i}\right)$.

Note that by definition a MR $r_{i}$ can only depend on itself if its defined type $t_{i}$ is employed for typing elements in the application condition $c_{L_{i}}$.

The SMRs for the SGDN for the social network query of Example 2 are depicted in Figure 4. We use here and in the following the more compact notation for SMRs where all graphs including the PACs and NACs are embedded into a single one 

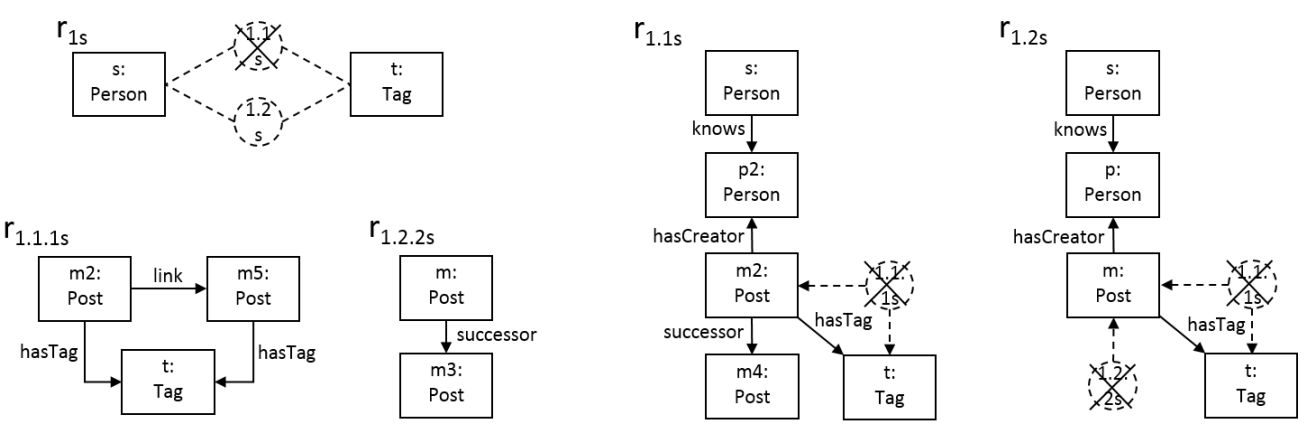

Figure 4: SMRs for the SGDN of the social network example

as presented in Figure 3 (c), moreover the RHS as well as the NAC equal to $p_{i}$ are omitted since they can be reconstructed from the rule's LHS uniquely.

Based on the previously introduced MRs or SMRs to encode the behavior of the network nodes of a GDN, we can now introduce our form of GDN or SGDN.

Definition $7\left(\mathrm{GDN}, \mathrm{SGDN}, \mathcal{L}_{G D N}, \mathcal{L}_{S G D N}\right)$. Given a finite graph $L$ typed over $T G$ and a GTS $\left(\mathcal{R}=\left(r_{i}\right)_{i \in I}, T G^{\prime}\right)$ of (simple) marking rules typed over the marking type graph $T G^{\prime}$ for $\left(L_{i}\right)_{i \in I}$, then $g d n_{L}=\left(\left(\mathcal{R}, T G^{\prime}\right), \rightsquigarrow_{d}^{+}\right)$is a (simple) generalized discrimination network over $L$ if the following conditions hold: (1) the transitive closure $\rightsquigarrow_{d}^{+}$is acyclic, (2) there is a unique so-called terminal rule $r_{t}$ with $L H S L_{t}=L$ for some $t \in I$, and (3) $\forall i \in I$ s.t. $i \neq t$ it holds that $\left(r_{t}, r_{i}\right)$ is in $\rightsquigarrow_{d}^{+}$. The graph query language $\mathcal{L}_{G D N}$

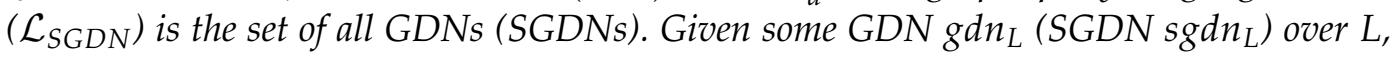
$L$ represents the so-called request graph.

Note that it follows directly from this definition that no rule of the GDN transitively depends on the terminal rule otherwise the transitive closure of the dependency relation would contain a cycle.

An example for a SGDN is depicted in Figure I (a) and 4, where Figure I (a) shows the dependencies between the nodes and Figure 4 shows the rules for the nodes $r_{1 s}, r_{1.1 s}, r_{1.2 s}, r_{1.1 .1 s}$, and $r_{1.2 .2 s}$.

In the following definitions we assume an operational query in the form of a GDN. In particular, each GDN represents a GTS with priorities. We consider each graph reachable via the GDN to encode an intermediate query result and the terminal graph then encodes the final query result. As shown in the subsequent lemma this terminal graph is indeed unique.

Lemma 1 (unique terminal graph). Given a $G D N g d n_{L}=\left(\left(\mathcal{R}, T G^{\prime}\right), \rightsquigarrow_{d}^{+}\right)$for $L$ typed over TG, then TERM $\left(g d n_{L}, G\right)$ consists of exactly one graph.

Proof. (sketch; more details see Section A.I) As there is an upper bound on matches that can be marked and rule applications always add exactly one such marking, 
$g d n_{L}$ terminates. As the priorities expressed by $\rightsquigarrow_{d}^{+}$exclude conflicting applications of different rules and acyclicity of $\rightsquigarrow_{d}^{+}$excludes conflicting applications of a rule with itself, $g d n_{L}$ is also confluent.

Definition 8 (ans $\left.s_{G D N}\right)$. Given the graph query language $\mathcal{L}_{G D N}$, the answer set mapping ans $_{G D N}$ for $\mathcal{L}_{G D N}$ is given by

$$
\operatorname{ans}_{G D N}\left(g d n_{L}, G\right):=\left\{o: L \rightarrow G \mid G_{i} \Rightarrow_{o^{\prime}, r_{t}} G_{i}^{\prime} \text { is a direct } G T \text { in } t \wedge o(L)=o^{\prime}(L)\right\}
$$

with $g d n_{L}=\left(\left(\mathcal{R}, T G^{\prime}\right), \rightsquigarrow_{d}^{+}\right)$some GDN such that $L$ is typed over $T G, G$ a graph in $\mathcal{L}(T G), r_{t}$ the terminal rule of $g d n_{L}$ and $t: G \Rightarrow_{g d n_{L}}^{*} G^{\prime}$ some transformation with $\left\{G^{\prime}\right\}=\operatorname{TERM}\left(g d n_{L}, G\right)$.

The above definition is well-defined, since matches are never destroyed because of dealing only with non-deleting rules and no conflicting direct transformations arise because of the priorities encoded with $\rightsquigarrow_{d}^{+}$and acyclicity of $\rightsquigarrow_{d}^{+}$(as mentioned also w.r.t. terminal graph uniqueness). Moreover, for $o^{\prime}: L \rightarrow G_{i}$ it holds that $o^{\prime}(L)$ is a subgraph of $G$.

In practice, it is important for efficiency reasons that we can reconstruct the answer set $a n s_{G D N}\left(g d n_{L}, G\right)$ from the markings in the terminal graph $G^{\prime}$ without having to consider the transformation $t$ leading to $G^{\prime}$. Under the condition that we only query graphs without parallel edges of the same type this can be done uniquely (see Section A.4).

The following result shows that for each SGDN an equivalent tree-like SGDN exists in which no two rules exist that directly depend on the same rule and each dependency is caused by exactly one PAC/NAC. As the considerations in the following section are considerably simpler when operating on tree-like SGDNs, we will w. l.o.g. (cf. Lemma 2) in the following restrict to tree-like networks.

Definition 9 (SGDT, $\mathcal{L}_{S G D T}$ ). A simple generalized discrimination tree (SDGT) is a SGDN sgdn $n_{L}=\left(\left(\mathcal{R}=\left(r_{i}\right)_{i \in I}, T G^{\prime}\right), \rightsquigarrow_{d}^{+}\right)$such that (1) for each $\left(r_{i}, r_{j}\right) \in \rightsquigarrow_{d}$ no $k \in I$ with $k \neq i$ exists s.t. $\left(r_{k}, r_{j}\right) \in \rightsquigarrow_{d}$ and (2) for each $i \in I$ it holds that for each PAC or NAC of $r_{i}$ no other PAC or NAC in $r_{i}$ exists referring to the same marking node type. The graph query language $\mathcal{L}_{S G D T}$ is the set of all SGDTs.

Lemma $2\left(\mathcal{L}_{S G D N} \sim \mathcal{L}_{S G D T}\right)$. Given a $S G D N$ sgdn $n_{L}$ for a graph $L$ typed over $T G$, then it holds that a SGDT sgdt $t_{L}$ exists such that $s g d n_{L} \sim \operatorname{sgdt} t_{L}$. Moreover, $\mathcal{L}_{S G D N} \sim \mathcal{L}_{S G D T}$.

Proof. (sketch, details see Section A.2) We can show by induction over the depth of $\rightsquigarrow_{d}^{+}$that we can construct an equivalent tree by employing copied rules with disjoint markings. Since each SGDT is in particular also a SGDN, it directly follows that $\mathcal{L}_{S G D N} \sim \mathcal{L}_{S G D T}$. 


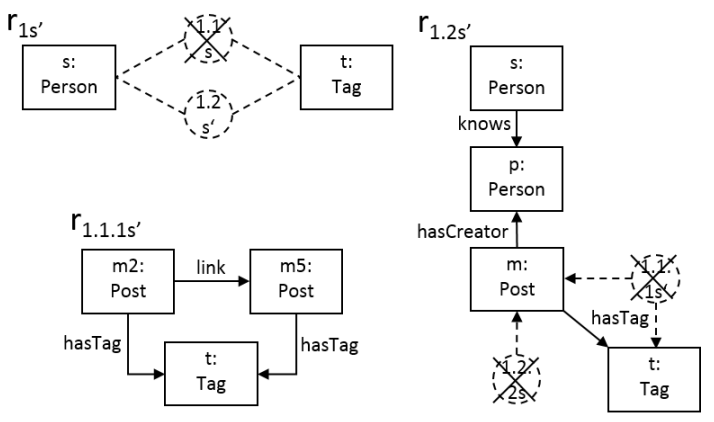

(a)
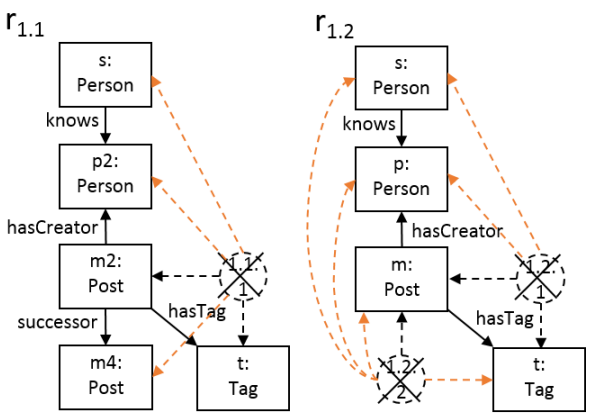

(b)

Figure 5: SMRs for the SGDT for the social network example (a) and with maximal context (b) as denoted by the orange dashed lines

The SMRs of the SGDT related to the SGDN of Figure 1 (a) depicted in Figure 1 (b) where multiple referenced SMRs are simply replicated are presented in Figure 5 (a). The rules $r_{1.1 s}, r_{1.1 .1 s}$, and $r_{1.2 .2 s}$ of Figure 4 are not shown in Figure 5 since they remain the same. Rules $r_{1 s^{\prime}}$ and $r_{1.2 s^{\prime}}$, which differ from the rules $r_{1 s}$ and $r_{1.2 s}$ of Figure 4 only concerning the referenced other rules are shown, along with rule $r_{1.1 .1 s^{\prime}}$, which is a replication of rule $r_{1.1 .1 s}$ that differs only w. r. t. created elements (omitted from the visualization).

\section{Equivalence to Nested Graph Conditions}

In order to prove that each NGC can be represented by some equivalent SGDT, we first show in the following Lemmas that the standard operators in NGCs (true, existential quantification, negation and binary conjunction) (Def. see Sect. 2) can be simulated by equivalent constructions in a SGDT.

Lemma 3 (true). Given the NGC true over $L$, there exists some SGDT sgdt $t_{L}$ such that $\operatorname{sgdt}_{L} \sim$ true.

Proof. Let $\left.s g d t_{L}=\left(\left\{r_{L, \text { true }}\right\}, T G^{\prime}\right), \rightsquigarrow_{d}^{+}\right)$for $L$ typed over $T G$ with marking rule $r_{L, t r u e}=\langle p: L \rightarrow R, \nexists p\rangle$, then for each graph $G$ typed over $T G, a n s_{G D N}\left(\operatorname{sgdt} t_{L}, G\right)$ consists of all morphisms $p: L \rightarrow G$. This means that $s g d t_{L} \sim$ true.

Lemma $4\left(\exists\left(a: L \rightarrow L^{\prime}, c_{L^{\prime}}\right)\right)$. Given some NGC $\exists\left(a: L \rightarrow L^{\prime}, c_{L^{\prime}}\right)$ and SGDT sgdt $t_{L^{\prime}}^{\prime}$ such that sgdt $L_{L^{\prime}}^{\prime} \sim c_{L^{\prime}}$, there exists some SGDT sgdt $t_{L}$ such that $\operatorname{sgdt}_{L} \sim \exists(a: L \rightarrow$ $\left.L^{\prime}, c_{L^{\prime}}\right)$. 
Proof. Suppose that $s g d t_{L^{\prime}}^{\prime}$ has the terminal rule $r_{t}^{\prime}=\left\langle p_{t}^{\prime}: L^{\prime} \rightarrow R^{\prime}, \nexists p_{t}^{\prime} \wedge c_{L^{\prime}}^{\prime}\right\rangle$. We construct the SGDT $s g d t_{L}$ from $s g d t_{L^{\prime}}^{\prime}$ by adding as terminal rule the rule $r_{L, \exists a}=\left\langle p: L \rightarrow R, \nexists p \wedge \exists\left(p_{t_{\mid a(L)}}^{\prime} \circ a^{\prime}\right.\right.$, true $\left.)\right\rangle$ with $a^{\prime}: L \rightarrow a(L)$ such that $a^{\prime}$ is identical with $a$. Consider $\operatorname{ans}_{G D N}\left(\operatorname{sgdt}_{L}, G\right)$ consisting of all morphisms $o: L \rightarrow G$ s.t. $r_{L, \exists a}$ created a marking to $o(L)$. Because of the PAC $\exists\left(p_{t_{\mid a(L)}^{\prime}}^{\prime} \circ a^{\prime} \text {, true }\right)^{3}$ in the terminal rule $r_{L, \exists a}$ this can only be the case if $r_{t}^{\prime}$ created a marking for some $o^{\prime}\left(L^{\prime}\right)$ with $o^{\prime}: L^{\prime} \rightarrow G$ a morphism in $\operatorname{ans}_{G D N}\left(\operatorname{sgdt}_{L^{\prime}}^{\prime}, G\right)$. Since $s g d t_{L^{\prime}}^{\prime} \sim c_{L^{\prime}}$ we know that $r_{t}^{\prime}$ created a marking to $o^{\prime}\left(L^{\prime}\right)$ iff $o^{\prime} \models c_{L^{\prime}}$. Therefore we conclude that $o=\exists\left(a: L \rightarrow L^{\prime}, c_{L^{\prime}}\right)$ and thus $\operatorname{sgdt}_{L} \sim \exists\left(a: L \rightarrow L^{\prime}, c_{L^{\prime}}\right)$.

Lemma $5\left(\neg c_{L}\right)$. Given some NGC $\neg c_{L}$ and SGDT $\operatorname{sgdt}_{L}^{\prime}$ such that $\operatorname{sgdt}_{L}^{\prime} \sim c_{L}$, there exists some SGDT sgdt such that $\operatorname{sgdt}_{L} \sim \neg c_{L}$.

Proof. Suppose that $s g d t_{L}^{\prime}$ has the terminal rule $r=\left\langle p^{\prime}: L \rightarrow R^{\prime}, \nexists p^{\prime} \wedge c_{L}^{\prime}\right\rangle$. Then consider the SGDT $s g d t_{L}$ having an additional rule $r_{L,\urcorner}=\langle p: L \rightarrow R, \nexists p \wedge$ $\left.\nexists p^{\prime}\right\rangle$ w.r.t. $s g d t_{L}^{\prime}$ as terminal rule. Consider $\operatorname{ans}_{G D N}\left(s g d t_{L}, G\right)$ consisting of all morphisms $o: L \rightarrow G$ s.t. $r_{L,\urcorner}$ created a marking to $o(L)$. Because of the NAC $\nexists p^{\prime}$ in the terminal rule $r_{L, \neg}$ this can only be the case if $r$ did not create a marking to $o(L)$. Since $s g d t_{L}^{\prime} \sim c_{L}$ we know that $r$ created a marking to $o(L)$ iff $o \models c_{L}$. Therefore we conclude that $o=\neg c_{L}$ and thus $\operatorname{sgdt}_{L} \sim \neg c_{L}$.

Lemma $6\left(c_{1, L} \wedge c_{2, L}\right)$. Given some NGC $c_{1, L} \wedge c_{2, L}$ and SGDTs sgdt $t_{L}^{1}$ and $\operatorname{sgdt}_{L}^{2}$ such that $\operatorname{sgdt}_{L}^{1} \sim c_{1, L}$ and $\operatorname{sgdt} t_{L}^{2} \sim c_{2, L}$, there exists some SGDT $\operatorname{sgdt}_{L}$ such that $\operatorname{sgdt}_{L} \sim$ $c_{1, L} \wedge c_{2, L}$.

Proof. Let $r_{1}=\left\langle p_{1}: L \rightarrow R_{1}, \nexists p_{1} \wedge c_{L}\right\rangle$ and $r_{2}=\left\langle p_{2}: L \rightarrow R_{2}, \nexists p_{2} \wedge c_{L}^{\prime}\right\rangle$ be the terminal rules for $s g d t_{L}^{1}$ and $s g d t_{L}^{2}$, respectively. Consider the SGDT $s g d t_{L}$ consisting of the subtrees $s g d t_{L}^{1}$ and $s g d t_{L}^{2}$ with the additional rule $r_{L, \wedge}=\langle p: L \rightarrow$ $\left.R, \nexists p \wedge \exists p_{1} \wedge \exists p_{2}\right\rangle$ as terminal rule. Consider $a n s_{G D N}\left(\operatorname{sgdt}_{L}, G\right)$ consisting of all morphisms $o: L \rightarrow G$ s.t. $r_{L, \wedge}$ created a marking to $o(L)$. Because of the PACs $\exists p_{1}$ and $\exists p_{2}$ in the terminal rule $r_{L, \wedge}$ this can only be the case if $r_{1}$ as well as $r_{2}$ created a marking to $o(L)$. Since $s g d t_{L}^{1} \sim c_{1, L}$ resp. $s g d t_{L}^{2} \sim c_{2, L}$ we know that $r_{1}$ resp. $r_{2}$ created a marking to $o(L)$ iff $o=c_{1, L}$ resp. $o=c_{2, L}$. Therefore we conclude that $o \mid=c_{1, L} \wedge c_{2, L}$ and thus $s g d t_{L} \sim c_{1, L} \wedge c_{2, L}$.

Now we can prove that each NGC can be emulated by an equivalent SGDT.

3In [5] we had the PAC $\exists\left(p_{t}^{\prime} \circ a^{\prime}\right.$, true $)$ violating the restricted form of PACs allowed in a simple marking rule. This slightly corrected but in this case equivalent version of the PAC restricts the codomain of $a$ and the domain of $p_{t}^{\prime}$ such that exactly one marking node together with marking edges referring to $a^{\prime}(L)=a(L)$ are required. 
Proposition 1 (emulate NGC by SGDT). Given a NGC $c_{L}$, there exists a SGDT sgdt s.t. $\operatorname{sgdt}_{L} \sim c_{L}$.

Proof. We prove this by induction over the nesting level of NGCs and the way they are constructed.

Base case: By Lemma 3 it follows that for $c_{L}=$ true with nesting level o an equivalent SGDT with a single marking rule exists. From Lemma 5 and 6 it follows that for any combination of conditions of nesting level o we can still construct an equivalent SGDT.

Induction step: By Lemma 4 and the induction hypothesis it follows that for any condition $\exists\left(a: L \rightarrow L^{\prime}, c_{L^{\prime}}\right)$ of nesting level $n+1$ it follows that an equivalent SGDT exists. From Lemma 5 and 6 it follows that for any combination of conditions of nesting level $n+1$ we can still construct an equivalent SGDT.

We still need to show that also each SGDT can be emulated by an equivalent NGC. An important first step thereby is the construction of a transformation of some SGDT into a SGDT with so-called maximal context. Marking rules in GDNs are able to pass merely the context necessary for the next subquery, which is a practical property for efficiency reasons, but not for showing equivalence with NGCs based on maximal context passing. With context propagation we therefore introduce a mechanism transforming marking rules passing only partial context into rules passing maximal context. We moreover show that this context propagation does not alter the answer set semantics of the corresponding SGDT.

Definition 10 (maximal context). Given a SGDT sgdt $t_{L}$ for a graph $L$ typed over $T G$ then $\operatorname{sgdt}_{L}$ has maximal context if for each two SMRs $r_{i}=\left\langle p_{i}: L_{i} \rightarrow R_{i}, \nexists p_{i} \wedge \wedge_{j \in J_{i}}\left(\exists p_{j}^{i}\right.\right.$ : $\left.\left.L_{i} \rightarrow P_{j}^{i}\right) \wedge \bigwedge_{k \in K_{i}}\left(\nexists n_{k}^{i}: L_{i} \rightarrow N_{k}^{i}\right)\right\rangle$ and $r_{l}=\left\langle p_{l}: L_{l} \rightarrow R_{l}, \nexists p_{l} \wedge \bigwedge_{j \in J_{l}}\left(\exists p_{j}^{l}: L_{l} \rightarrow\right.\right.$ $\left.\left.P_{j}^{l}\right) \wedge \bigwedge_{k \in K_{l}}\left(\nexists n_{k}^{l}: L_{l} \rightarrow N_{k}^{l}\right)\right\rangle$ with marking node $l$ s.t. $\left(r_{i}, r_{l}\right) \in \rightsquigarrow_{d}$ because for some $j \in J_{i}$ (or $k \in K_{i}$ ) $p_{j}^{i}$ (or $n_{k}^{i}$, resp.) uses a type equal to the type $t_{l}$ of $l$, the sets $V_{j}^{i}$ (or $V_{k^{\prime}}^{i}$ resp.) constructed as follows are empty:

$$
\begin{gathered}
V_{j}^{i}=\left\{n \mid n \in L_{i}^{V} \text { s.t. } \nexists e \in\left(P_{j}^{i}\right)^{E} \text { with type of } s^{P_{j}^{i}}(e)=t_{l} \wedge t^{P_{j}^{i}}(e)=p_{j}^{i}(n)\right\} \\
V_{k}^{i}=\left\{n \mid n \in L_{i}^{V} \text { s.t. } \nexists e \in\left(N_{k}^{i}\right)^{E} \text { with type of } s^{N_{k}^{i}}(e)=t_{l} \wedge t^{N_{k}^{i}}(e)=n_{k}^{i}(n)\right\}
\end{gathered}
$$

Lemma 7 (context propagation). Given a SGDT sgdt $t_{L}$ for a graph $L$ typed over TG with two rules $r_{i}$ and $r_{l}$ such that $\left(r_{i}, r_{l}\right) \in \rightsquigarrow_{d}$ with non-empty $V_{j}^{i}$ (or $V_{k}^{i}$ ) (as given in Def. IO), then there exists some $s g d t_{L}^{c}$ in which $\left(r_{i}, r_{l}\right)$ has been replaced by a SGDT with maximal context such that $s g d t_{L}^{c} \sim \operatorname{sgdt} t_{L}$.

Proof. (sketch; details see Lemma 7) We construct a $s g d t_{L}^{c}$ in which marking rules with propagated context check in contrast to $r_{l}$ the presence of additional nodes 
and edges in the queried graph $G$ that would otherwise have been searched for anyway by rule $r_{i}$ after all matches for $r_{l}$ had been found. Marking these elements earlier does not change the overall answer set.

Lemma 8 (maximal context). For a SGDT $s g d t_{L}$ for a graph L typed over TG their exists a SGDT sgdt $t_{L}^{\prime}$ with maximal context such that $s g d t_{L}^{\prime} \sim \operatorname{sgdt_{L}}$.

Proof. We proof this lemma by induction on the height of the tree.

Base case: Suppose that we have $s g d t_{L}$ with height $o$, then it trivially holds that $s g d t_{L}$ has maximal context already.

Induction step: Suppose that we have $s g d t_{L}$ with height $n+1$. Then apply subsequently for each $\left(r_{t}, r_{i}\right) \in \rightsquigarrow_{d}$ context propagation to $s g d t_{L}$ obtaining according to Lemma 7 an equivalent $s g d t_{L}^{c}$ of height $n+1$. Now consider for each $r_{i}$ the subtree $s g d t_{L_{i}^{c}}^{r_{i}}$ in $s g d t_{L}^{c}$ of height $n$. Then for each $s g d t_{L_{i}^{c}}^{r_{i}}$ by induction hypothesis an equivalent SGDT $s g d t_{L_{i}^{c}}^{\prime}$ with maximal context exists. Replacing in $s g d t_{L}^{c}$ each $s g d t_{L_{i}^{c}}^{r_{i}}$ with $s g d t_{L_{i}^{c}}^{\prime}$ we obtain a SGDT $s g d t_{L}^{\prime}$ with maximal context s.t. $s g d t_{L}^{\prime} \sim s g d t_{L}$.

Two of the modified SMRs of the SGDT depicted in Figure 1 (c) with maximal context related to the SGDN of Figure 1 (a) are presented in Figure 5 (b). While the rules $r_{1.1}$ and $r_{1.2}$ already have maximal context and therefore differ from the $r_{1.1 s}$ and $r_{1.2 s^{\prime}}$ only concerning the referenced other rules and additional links to bind the propagated context as depicted in Figure 5 (b) by the orange edges, the rules $r_{1.1 .1}, r_{1.2 .1}$, and $r_{1.2 .2}$ are extended with propagated context concerning the rules $r_{1.1 .1 s}, r_{1.1 .1 s^{\prime}}$, and $r_{1.2 .2 s}$ and in addition have to reference the new rules.

Now we are ready to prove that for each SGDT there exists an equivalent NGC and consequently also that the languages $\mathcal{L}_{S G D T}$ and $\mathcal{L}_{N G C}$ are equivalent.

Proposition 2 (emulate SGDT by NGC). Given, a SGDT $s g d t_{L}$ for a graph L typed over TG, then there exists a NGC $c_{L}$ s.t. $\operatorname{sgdt}_{L} \sim c_{L}$.

Proof. Because of Lemma 8 we can assume w. l.o.g. that $s g d t_{L}$ has maximal context. We perform the proof by induction on the height of the tree.

Base case: If $s g d t_{L}$ has height $\mathrm{o}$, then it consists merely of some terminal rule without any PACs or NACs. Then $a n s_{g d n}\left(s g d t_{L}, G\right)$ consists of all matches of the terminal rule into $G$. If we choose $c_{L}$ equal to true over $L$ then it returns exactly the same set of morphisms s.t. $s g d t_{L} \sim c_{L}$.

Induction step: Suppose that $s g d t_{L}$ has height $n+1$ and that it has terminal rule $r=\left\langle p: L \rightarrow R, \nexists p \wedge \bigwedge_{j \in J}\left(\exists p_{j}: L \rightarrow P_{j}\right) \wedge \bigwedge_{k \in K}\left(\nexists n_{k}: L \rightarrow N_{k}\right)\right\rangle$. Then we have a subtree $s g d t_{L_{j}}$ and $s g d t_{L_{k}}$ for each $p_{j}$ and each $n_{k}$, respectively. Because of induction hypothesis it holds that for each $s g d t_{L_{j}}$ and $s g d t_{L_{k}}$ there exists an equivalent NGC $c_{L_{j}}$ and $c_{L_{k}}$, respectively. Since $s g d t_{L}$ has maximal context, we 
moreover know that there exist morphisms $l_{j}: L \rightarrow L_{j}$ and $l_{k}: L \rightarrow L_{k}$. Consider the NGCs $c_{L}^{j}=\exists\left(l_{j}, c_{L_{j}}\right)$ and $c_{L}^{k}=\nexists\left(l_{k}, c_{L_{k}}\right)$ such that $c_{L}=\wedge_{j \in J} c_{L}^{j} \wedge \wedge_{k \in K} c_{L}^{k}$. Now $\operatorname{ans}_{G D N}\left(\operatorname{sgdt}_{L}, G\right)$ for some $G$ consists of all morphisms $o: L \rightarrow G$ such that the terminal rule of each $s g d t_{L_{j}}$ and $s g d t_{L_{k}}$ has been applied and not been applied, respectively. The latter is equivalent with the fact that for each $j \in J$ a morphism $o_{j}: L_{j} \rightarrow G$ exists s.t. $o_{j} \circ l_{j}=o$ with $o_{j} \in a n s_{G D N}\left(\operatorname{sgdt}_{L_{j}}, G\right)=a n s_{N G C}\left(c_{L_{j}}, G\right)$. Analogously for each $k \in K$ there does not exist a morphism $o_{k}: L_{k} \rightarrow G$ s.t. $o_{k} \circ l_{k}=o$ and $o_{k} \in a n s_{G D N}\left(\operatorname{sgdt}_{L_{k}}, G\right)=a n s_{N G C}\left(c_{L_{k}}, G\right)$. This is exactly what also each morphism $o: L \rightarrow G$ in $\operatorname{ans}_{N G C}\left(c_{L}, G\right)$ needs to fulfill s.t. we can conclude that $\operatorname{sgdt}_{L} \sim c_{L}$.

Theorem 1 (language equivalence). $\mathcal{L}_{S G D N} \sim \mathcal{L}_{S G D T} \sim \mathcal{L}_{N G C}$

Proof. From Proposition 1 and 2 we can follow directly that $\mathcal{L}_{S G D T} \sim \mathcal{L}_{N G C \text {. From }}$ Lemma 2 we can conclude that $\mathcal{L}_{S G D N} \sim \mathcal{L}_{S G D T}$.

\section{Discussion}

In this section, we will discuss a more expressive variant, a minimal variant, as well as some observations and implications for optimization of graph queries and incremental updates concerning GDNs and the proposed SGDNs.

In particular, we can show that for minimal SGDT (MSGDT) - SGDT with at most two direct dependencies per SMR, where all rules adhere to one of the four rule schemes introduced in Lemmata 3, 4, 5, and 6, and where in addition all rules for existential quantification are limited to at most one additional element in form of a node or edge - holds that $\mathcal{L}_{M S G D T} \sim \mathcal{L}_{N G C}$ (see Section A.6) and thus the additional restrictions do not result in any loss of expressive power. As often the tree-like simplification is not wanted, we further name SGDN that are not MSGDT but fulfill all conditions besides the tree nature as MSGDN.

There are several approaches for optimization of graph queries or incremental updates of graph queries based on RETE networks (cf. [11]) such as [7] and VIATRA [4] that can be conceptually mapped to MSGDN. In these cases the RETE network structure supports only at most two direct dependencies like MSGDN and the computations of the nodes of the RETE network can be matched to the four permitted cases of MSGDN. Our results also indicate that these approaches have the same expressiveness as NGC.

In our own practical work on graph queries [6], we conceptually employ SGDN with marking rules in form of graph transformation rules for optimization of queries and incremental updates of graph queries. We were able to show that the 
more powerful capabilities of a single node (marking rule) and advanced dynamic pattern matching strategies [12] can lead to considerable improvements concerning the computation speed and memory consumption for SGDN compared to the restricted case of MSGDN (resp. RETE network). Similar results have been obtained also in the relational case where it has been shown that the more general GATOR networks can outperform RETE networks [14]. Consequently, it seems reasonable to study the broader class of SGDN for optimization of queries and incremental updates of graph queries and not more restricted forms such as MSGDN or MSGDT. In particular the context propagation (see Definition 10 ) and its inverse context elimination seem useful tools here to minimize the effort for subqueries and the propagation of their results in the network.

We can also have more expressive generalized discrimination networks as given in Def. 7 for which we can show (see Section A.5 that they will not lead to an increase of expressive power such that the language equivalence $\mathcal{L}_{G D N} \sim \mathcal{L}_{N G C}$ holds. In particular, the use of NGCs as application conditions in the marking rules results in much more complex direct dependencies. Thus the discussed increase in expressive power of the marking rules will not increase the expressive power of the discrimination network.

The study of more powerful marking rules may still be useful when efficient matching algorithms for the supported fragment of NGCs can be employed. It is to be noted, that in case of incremental updates for graph queries the limitation to simple graph rules seems necessary to be able to propagate update effects without the need to maintain expensive additional data structures to detect the need for updates (cf., for example, instance-based scopes in [8]), while in case of optimizations of graph queries this restriction may be not always helpful.

Furthermore the language equivalence $\mathcal{L}_{G D N} \sim \mathcal{L}_{N G C}$ only applies unless we leave the realm of pattern-based property specification concepts such as NGC and consider also path-related properties [17] or we permit cycles in the network in a controlled manner as in our own practical work on graph queries [6] to be able to support path-related properties (analogously to the controlled and repeated rule applications to support path-related properties used in [3]).

\section{Conclusion and Future Work}

Analog to the relational database case where the relational calculus and generalized discrimination networks have been studied to find appropriate decompositions into subqueries and ordering of these subqueries for query evaluation or incremental updates of queries, we presented in this paper GDNs for graph queries with simple 
operational concepts where graph transformations describe the node behavior. We further showed that the proposed GDNs in different forms all have the same expressive power as NGC.

We plan to study in our future work the complexity of evaluating and updating SGDNs, their optimization, and possible extensions of SGDNs towards path-related properties to also formally cover our own practical work on graph queries [6] supporting cycles in the network.

\section{Acknowledgments}

This work was partially developed in the course of the project Correct Model Transformations II (GI 765/1-2), which is funded by the Deutsche Forschungsgemeinschaft.

We are grateful to Johannes Dyck for his contribution to our discussions and feedback to draft versions of the paper. 
On the Operationalization of Graph Queries with Generalized Discrimination Networks

\section{References}

[1] Serge Abiteboul, Richard Hull, and Victor Vianu, editors. Foundations of Databases: The Logical Level. Addison-Wesley Longman Publishing Co., Inc., Boston, MA, USA, Ist edition, 1995.

[2] Renzo Angles. A Comparison of Current Graph Database Models. In Proc. of the $28^{\text {th }}$ Int. Conf. on Data Engineering, pages 171-177. IEEE, April 2012.

[3] Basil Becker, Leen Lambers, Johannes Dyck, Stefanie Birth, and Holger Giese. Iterative Development of Consistency-Preserving Rule-Based Refactorings. In Jordi Cabot and Eelco Visser, editors, Theory and Practice of Model Transformations, Fourth International Conference, ICMT 2011, Zurich, Switzerland, June 27-28, 2011. Proceedings, volume 6707 of Lecture Notes in Computer Science, pages 123-137. Springer / Heidelberg, 2011.

[4] Gábor Bergmann, András Ökrös, István Ráth, Dániel Varró, and Gergely Varró. Incremental Pattern Matching in the VIATRA Model Transformation System. In Proceedings of the $3^{\text {rd }}$ International Workshop on Graph and Model Transformations, GRaMoT 'o8, pages 25-32. ACM, 2008.

[5] Thomas Beyhl, Dominique Blouin, Holger Giese, and Leen Lambers. On the Operationalization of Graph Queries with Generalized Discrimination Networks. In Rachid Echahed and Mark Minas, editors, Proceedings of the $9^{\text {th }}$ International Conference on Graph Transformations, pages 170-186. Springer, 2016.

[6] Thomas Beyhl and Holger Giese. Incremental View Maintenance for Deductive Graph Databases using Generalized Discrimination Networks. In Graphs as Models 2016. Electronic Proceedings in Theoretical Computer Science, 2016. to appear.

[7] H. Bunke, T. Glauser, and T.-H. Tran. An efficient implementation of graph grammars based on the RETE matching algorithm. In Hartmut Ehrig, HansJörg Kreowski, and Grzegorz Rozenberg, editors, Graph Grammars and Their Application to Computer Science, volume 532 of LNCS, pages 174-189. Springer, 1991.

[8] Alexander Egyed. Instant Consistency Checking for the UML. In ICSE 'o6: Proceedings of the 28th International Conference on Software Engineering, pages 381-390, Shanghai, China, 20-28 May 2006.

[9] Hartmut Ehrig, Karsten Ehrig, Ulrike Prange, and Gabriele Taentzer. Fundamentals of Algebraic Graph Transformation. Springer, 2006. 
[10] Hartmut Ehrig, Ulrike Golas, Annegret Habel, Leen Lambers, and Fernando Orejas. M-Adhesive Transformation Systems with Nested Application Conditions, Part 2: Embedding, Critical Pairs and Local Confluence. Fundamenta Informaticae, 118(1-2):35-63, 2012.

[11] Charles L. Forgy. Rete: A Fast Algorithm for the Many Pattern/Many object Pattern Match Problem. Artificial Intelligence, 19(1):17-37, 1982.

[12] Holger Giese, Stephan Hildebrandt, and Andreas Seibel. Improved Flexibility and Scalability by Interpreting Story Diagrams. In Tiziana Magaria, Julia Padberg, and Gabriele Taentzer, editors, Proc. of the 8th International Workshop on Graph Transformation and Visual Modeling Techniques, volume 18. Electronic Communications of the EASST, 2009.

[13] Annegret Habel and Karl-Heinz Pennemann. Correctness of high-level transformation systems relative to nested conditions. Mathematical Structures in Computer Science, 19:1-52, 2009.

[14] Eric N. Hanson, Sreenath Bodagala, and Ullas Chadaga. Trigger Condition Testing and View Maintenance Using Optimized Discrimination Networks. Transactions on Knowledge and Data Engineering, 14(2):261-280, Mar 2002.

[15] Huahai He and Ambuj K. Singh. Graphs-at-a-time: Query Language and Access Methods for Graph Databases. In Proceedings of the 2008 ACM SIGMOD International Conference on Management of Data, pages 405-418. ACM, 2008.

[16] Linked Data Benchmark Council, Coordinator: Arnau Prat (UPC). LDBC Social Network Benchmark (SNB) - First Public Draft Release vo.2.2, 2015. https://github.com/ldbc/ldbc_snb_docs/blob/master/LDBC_SNB_vo.2.2.pdf, accessed October 2016.

[17] Christopher M. Poskitt and Detlef Plump. Verifying monadic second-order properties of graph programs. In Proc. International Conference on Graph Transformation (ICGT 2014), volume 8571 of LNCS, pages 33-48. Springer, 2014.

[18] Peter T. Wood. Query Languages for Graph Databases. SIGMOD Rec., 41(1):5060, April 2012. 


\section{Appendix}

The following appendix presents omitted proofs and results as well as more details for the presented examples.

\section{A. Omitted Results and Proofs}

\section{A.1. Proof of Lemma田}

Proof of Lemma 1 . Given a GDN $g d n_{L}=\left(\left(\mathcal{R}, T G^{\prime}\right), \rightsquigarrow_{d}^{+}\right)$for $L$ typed over $T G$, then $\operatorname{TERM}\left(g d n_{L}, G\right)$ consists of exactly one graph.

Proof. Since each marking rule creates a node and is non-deleting it holds that the number of nodes is strictly monotonically increasing. Moreover, there is an upper bound on the nodes that can be created since for each marking rule $r_{i}=\left\langle p_{i}: L_{i} \rightarrow\right.$ $\left.R_{i}, \nexists p_{i} \wedge c_{L, i}\right\rangle$ the NAC $\nexists p_{i}$ excludes that the rule can be applied multiple times for the same match. Since we have non-deleting rules matches cannot be destroyed and having a finite number of potential matches we can follow that $g d n_{L}$ terminates.

Furthermore, two non-deleting GT rules $r_{i}$ and $r_{j}$ are in conflict if rule $r_{j}$ would be able to create elements that violate the application condition of $r_{i}$ (or vice versa) [10]. Considering $r_{i}, r_{j}$ to be marking rules this could only happen if $r_{j}$ creates marking elements referred to by $r_{i}$, which is only the case if $\left(r_{i}, r_{j}\right) \in \rightsquigarrow_{d}$ (or vice versa). For $i \neq j$ this would mean that by the priority mechanism $r_{i}$ and $r_{j}$ would not be applicable to the same graph in the first place. For $i=j$ we would have that $\left(r_{i}, r_{i}\right) \in \rightsquigarrow d$, which is in contradiction with acyclicity of $\rightsquigarrow_{d}^{+}$. Thus $g d n_{L}$ is confluent and also terminates with a unique result.

\section{A.2. Proof of Lemma 2}

Proof of Lemma 2. Given a SGDN $s g d n_{L}$ for a graph $L$ typed over $T G$, then it holds that a SGDT $s g d t_{L}$ exists such that $s g d n_{L} \sim \operatorname{sgdt} t_{L}$. Moreover, $\mathcal{L}_{S G D N} \sim \mathcal{L}_{S G D T}$.

Proof. We show the above lemma by induction over the depth of $\rightsquigarrow_{d}^{+}$for some given $\operatorname{sgdn_{L}}=\left(\left(\mathcal{R}, T G^{\prime}\right), \rightsquigarrow_{d}^{+}\right)$.

Base case: If the depth of $\rightsquigarrow_{d}^{+}$equals o $s g d n_{L}$ is trivially already a SGDT. Induction step: Suppose that $\rightsquigarrow_{d}^{+}$has depth $n+1$ and that $r=\left\langle p: L \rightarrow R, \nexists p \wedge \wedge_{j \in J}\left(\exists p_{j}\right.\right.$ : $\left.\left.L \rightarrow P_{j}\right) \wedge \wedge_{k \in K}\left(\nexists n_{k}: L \rightarrow N_{k}\right)\right\rangle$ is the terminal rule of $s g d n_{L}$. Then for each $r_{j}$ such that $\left(r, r_{j}\right) \in \rightsquigarrow_{d}$ consider the SGDN of depth n $\operatorname{sgdn_{L_{j}}^{r_{j}}}=\left(\left(\mathcal{R}_{r_{j}}, T G^{\prime}\right), \rightsquigarrow \underset{d, r_{j}}{+}\right)$ 
with $\mathcal{R}_{r_{j}}=\left\{r_{j}\right\} \cup\left\{r_{i} \mid\left(r_{j}, r_{i}\right) \in \rightsquigarrow_{d}^{+}\right\}$and $\rightsquigarrow_{d, r_{j}}^{+}=\left\{\left(r_{i}, r_{i}^{\prime}\right) \mid\left(r_{i}, r_{i}^{\prime}\right) \in \rightsquigarrow_{d}^{+} \wedge r_{i}, r_{i}^{\prime} \in \mathcal{R}_{r_{j}}\right\}$. We can now replace in $s g d n_{L}$ by induction hypothesis each SGDN $s g d n_{L_{j}}^{r_{j}}$ by an equivalent SGDT $s g d t_{L_{j}}^{r_{j}}$ obtaining the SGDN $s g d n_{L}^{\prime}$, which fulfills property (1), but not yet property (2) of SGDTs as given in Def. 9 . We still need to ensure that no two PACs/NACs of the terminal rule $r$ refer to identical marking node types. Thus consider for each $r_{j}$ such that $\left(r, r_{j}\right) \in \rightsquigarrow_{d}$ all PACs/NACs in $r$ referring to the marking node type $t_{j}$ of rule $r_{j}$. If there are at least two, then we build for each such PAC/NAC an equivalent copy of $s g d t_{L_{j}}^{r_{j}}$ with new disjoint marking types. We replace in $s g d n_{L}^{\prime}$ each subtree $s g d t_{L_{j}}^{r_{j}}$ by these copies and retype the PACs and NACs in the terminal rule $r$ accordingly. In this way we obtain an equivalent SGDT $s g d t_{L}$ from the SGDN $s g d n_{L}$.

Since each SGDT is in particular also a SGDN, it directly follows that $\mathcal{L}_{S G D N} \sim$ $\mathcal{L}_{S G D T}$.

\section{A.3. Proof of Lemma 7}

Proof of Lemma 7 Given a SGDT $s g d t_{L}$ for a graph $L$ typed over TG with two rules $r_{i}$ and $r_{l}$ such that $\left(r_{i}, r_{l}\right) \in \rightsquigarrow_{d}$ with non-empty $V_{j}^{i}$ (or $V_{k}^{i}$ ) (as given in Def. 10), then there exists some $s g d t_{L}^{c}$ in which $\left(r_{i}, r_{l}\right)$ has been replaced by a SGDT with maximal context such that $s g d t_{L}^{c} \sim s g d t_{L}$.

Proof. Given a SGDT $s g d t_{L}$ with marking rule set $\mathcal{R}=\left(r_{i}\right)_{i \in I}$ for a graph $L$ typed over TG with two SMRs $r_{i}=\left\langle p_{i}: L_{i} \rightarrow R_{i}, \nexists p_{i} \wedge \bigwedge_{j \in J_{i}}\left(\exists p_{j}^{i}: L_{i} \rightarrow P_{j}^{i}\right) \wedge \bigwedge_{k \in K_{i}}\left(\nexists n_{k}^{i}:\right.\right.$ $\left.\left.L_{i} \rightarrow N_{k}^{i}\right)\right\rangle$ and $r_{l}=\left\langle p_{l}: L_{l} \rightarrow R_{l}, \nexists p_{l} \wedge \bigwedge_{j \in J_{l}}\left(\exists p_{j}^{l}: L_{l} \rightarrow P_{j}^{l}\right) \wedge \bigwedge_{k \in K_{l}}\left(\nexists n_{k}^{l}: L_{l} \rightarrow\right.\right.$ $\left.\left.N_{k}^{l}\right)\right\rangle$ with marking node $l$ s.t. $\left(r_{i}, r_{l}\right) \in \rightsquigarrow_{d}$ because for some $j \in J_{i}$ (or $k \in K_{i}$ ) $p_{j}^{i}$ (or $n_{k}^{i}$, resp.) uses a type equal to $t_{l}$, we first introduce the construction of the SGDT $\operatorname{sgdt}_{L}^{c}$. Now $r_{i, j}^{c}\left(r_{i, k}^{c}\right)$ equals a rule $r_{i}$, where $\exists p_{j}^{i}: L_{i} \rightarrow P_{j}^{i}\left(\right.$ or $\left.\nexists n_{k}^{i}: L_{i} \rightarrow N_{k}^{i}\right)$ has been replaced by the disjunction of $\exists p_{j}^{i, c}: L_{i} \rightarrow P_{j}^{i, c}$ (or conjunction of $\nexists n_{k}^{i, c}: L_{i} \rightarrow N_{k}^{i, c}$ ) for all possible $p_{j}^{i, c}$ (or $n_{k}^{i, c}$ ) with $P_{j}^{i, c}$ (or $N_{k}^{i, c}$ ) equal to $P_{j}^{i}\left(\right.$ or $N_{j}^{i}$ ) s.t. the unique node with node type $t_{l}$ holds in addition one outgoing edge for each node in $p_{j}^{i, c}\left(V_{j}^{i}\right)\left(n_{k}^{i, c}\left(V_{k}^{i}\right)\right)$, respectively. Moreover, consider each $r_{l, j}^{c}$ constructed from $r_{l}, p_{j}^{i}$ and $M\left(P_{j}^{i}\right)$ being the graph of $P_{j}^{i}$ consisting of the marking node $l$ and all outgoing edges with incident nodes in $L_{i}$ according to the following diagram s.t. all diagrams commute, $e$ and $e^{\prime}$ are jointly epimorphic, (1) is a pushout constructed as a pushout 
complement from $e$ and $p_{l}$ and (2) is a pushout constructed from $p$ and $i^{\prime \prime}$ :

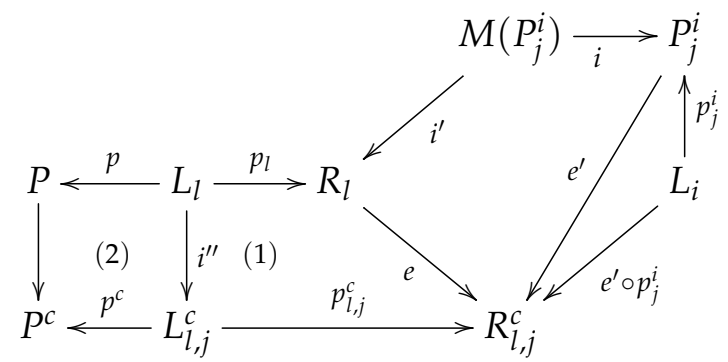

In this diagram the plain rule morphism of $r_{l, j}^{c}$ equals $p_{l, j}^{c}$ and each PAC (or NAC) from $r_{l}$ depicted by $p$ is translated into a corresponding equivalent PAC (or NAC) $p^{c}$ for $r_{l, j}^{c}$ by building PO (2). Analogously, each $r_{l, k}^{c}$ is constructed from $r_{l}, n_{k}^{i}$ and $M\left(N_{k}^{i}\right)$. Note that this diagram defines a unique monomorphism $\left(p_{l, j}^{c}\right)^{-1} \circ e^{\prime} \circ p_{j}^{i}$ from $L_{i}$ into $L_{l, j}^{c}$.

Let $g d t_{L}^{c}$ now be the GDT obtained from $s g d t_{L}$ by replacing $\left(r_{i}, r_{l}\right)$ by all pairs $\left(r_{i, j}^{c}, r_{l, j}^{c}\right)$ (or $\left(r_{i, k}^{c}, r_{l, j}^{c}\right)$, resp.). Now SGDT $s g d t_{L}^{c}$ is the SGDT that can be obtained from the GDT $g d t_{L}^{c}$ by transforming according to Proposition 1 the application condition with the disjunction (not adhering to the SMR scheme) in $r_{i, j}^{c}$ into a SGDT and replacing it by a simple PAC referring to the terminal rule of the corresponding tree.

By construction in $s g d t_{L}^{c}$ the marking rules $r_{l, j}^{c}\left(\right.$ or $r_{l, k}^{c}$ ) check in contrast to $r_{l}$ the presence of additional nodes and edges in the queried graph $G$ that would otherwise have been searched for later by rule $r_{i}$ after all matches for $r_{l}$ had been found. Marking these elements earlier does not change the overall answer set.

\section{A.4. From Marking Nodes to Graph Morphisms}

In practice, it is important for efficiency reasons that we can reconstruct the answer set $a n s_{G D N}\left(g d n_{L}, G\right)$ from the markings in the terminal graph $G^{\prime}$ without having to consider the transformation $t$ leading to $G^{\prime}$. In particular, we want to reconstruct where in $G^{\prime}$ the terminal rule has been matched without having to know in which direct transformation in $t$ this happened. In the following lemma we argue that this is possible if in the queried graph $G$ no parallel edges with the same type occur. Having parallel edges with the same type in $G$ can be emulated by replacing these edges by a node with two outgoing edges describing the source and target mapping of the edge.

Lemma 9 (extracting query result from markings). Given a graph $G$ typed over $T G$ without parallel edges of the same type and a GDN $g d n_{L}=\left(\left(\mathcal{R}, T G^{\prime}\right), \rightsquigarrow_{d}^{+}\right)$for a 
graph $L$ typed over TG such that $\left\{G^{\prime}\right\}=\operatorname{TERM}\left(g d n_{L}, G\right)$, then $a \operatorname{ans}_{G D N}\left(g d n_{L}, G\right)$ can be reconstructed uniquely from $G^{\prime}$.

Proof. We know that for a transformation $t: G \Rightarrow_{g d n_{L}}^{*} G^{\prime}$ and the terminal rule $r_{t}$ for $g d n_{L}$ with marking node $t$ it holds that

$$
\operatorname{ans}_{G D N}\left(g d n_{L}, G\right)=\left\{o: L \rightarrow G \mid G_{i} \Rightarrow_{o^{\prime}, r_{t}} G_{i}^{\prime} \text { is a direct GT in } \mathrm{t} \wedge o(L)=o^{\prime}(L)\right\} .
$$

In particular, each marking edge $e$ from a marking node $n$ of type $t$ in $G^{\prime}$ points uniquely to a node in $o^{\prime V}\left(L^{V}\right)$ because of the unique typing of $e$ w. r. t. nodes in $L^{V}$. The corresponding edge mapping $o^{\prime E}: L^{E} \rightarrow G_{i}^{\prime E}$ can be reconstructed by looking up in $G^{\prime}$ the edges in $L$ between the already found and uniquely marked source and target nodes in $G^{\prime}$ by comparing edge types. Altogether, this leads to a unique reconstruction of each $o: L \rightarrow G$, since no parallel edges in $G$ exist with the same type.

\section{A.5. $\mathcal{L}_{G D N} \sim \mathcal{L}_{N G C}$}

More expressive generalized discrimination networks can be defined by allowing the marking rules to be more expressive. A natural candidate for application conditions here are NGCs as employed for non-simple GDNs in Definition 7 . which result in much more complex direct dependencies. However, as we can show in the following, the discussed increase in expressive power of the marking rules will not increase the expressive power of the discrimination network 4

Theorem 2 (no increase of expressive power). $\mathcal{L}_{G D N} \sim \mathcal{L}_{N G C}$

Proof. (sketch) Each GDN can be transformed into an equivalent SGDN employing Proposition 1 for the application condition of each MR. Each SGDN is obviously a GDN such that $\mathcal{L}_{G D N} \sim \mathcal{L}_{S G D N}$. Since we know also that $\mathcal{L}_{S G D N} \sim \mathcal{L}_{N G C}$ the above statement follows.

\section{A.6. $\mathcal{L}_{M S G D T} \sim \mathcal{L}_{N G C}$}

We can investigate which additional restrictions to SGDTs can be applied without loosing any expressive power.

\footnotetext{
4It has, however, to be noted that in case more expressive application conditions than nested graph conditions (e.g., path-related conditions) are considered, the resulting expressive power of the generalized discrimination network is increased as well.
} 
Definition $11\left(M S G D T, \mathcal{L}_{M S G D T}\right)$. A minimal simple generalized discrimination tree (MSGDT) is a SGDT where each simple marking rule adheres to one of the four rule schemes $r_{L, \text { true }}, r_{L, \exists a}, r_{L, \neg}$, or $r_{L, \wedge}$ introduced in Lemmata 3. 4, 5. and 6, respectively. In addition, the rule for existential quantification $r_{L, \exists a}$ is such that the codomain of a holds at most one additional node or edge w.r.t. a $(L)$. The graph query language $\mathcal{L}_{M S G D T}$ is the set of all MSGDTs.

Lemma 10 (emulate GDN by MSGDT). For each GDN there exists an equivalent MSGDT.

Proof. From Theorem 2 we know that for each GDN there exists some equivalent NGC. Each NGC can be transformed into an equivalent NGC using merely binary conjunction, negation, and existential quantification limited to at most one additional node or edge. In Proposition 1 and the according Lemmas it is shown that for this NGC an equivalent SGDT exists with equivalent restrictions such that in particular it is an MSGDT.

Lemma 11 (no further simplification). Any simplification w. r.t. the form of MSGDTs leads to a class of GDNs that is too weak to be equivalent with $\mathcal{L}_{N G C}$.

Proof. (sketch) We systematically look for all possible simplifications and show that they are not possible without loosing expressive power: a) The basic rule $r_{L \text {,true }}$ must be supported, as no simpler form of marking rule is possible. b) The existential quantification rule $r_{L, \exists a}$ must be permitted otherwise if additional nodes/edges are not permitted, the case of a NAC is not covered. c) The negation rule $r_{L,\urcorner}$ must be allowed, otherwise expressiveness would equal the match finding for one large graph as merely all PACs could be combined. This case would clearly not cover e. g. NACs and it is thus too weak to cover NGCs. d) The conjunction rule $r_{L, \wedge}$ must be permitted, otherwise if only a sequence of dependencies rather than binary trees are permitted, the case of an or (resp. $\neg((\neg a) \wedge(\neg b))$ ) cannot be covered and thus NGCs would not be covered either.

Theorem 3 (expressiveness of MSGDTs). $\mathcal{L}_{M S G D T} \sim \mathcal{L}_{N G C}$

Proof. According to Lemma 10 each GDN can be transformed into some equivalent MSGDT. Since each MSGDT is also a GDN it follows that $\mathcal{L}_{G D N} \sim \mathcal{L}_{M S G D T}$. We know that $\mathcal{L}_{G D N} \sim \mathcal{L}_{N G C}$ such that the above statement follows. 


\section{B. Complete Example}

This section presents in detail the complete complex query example of this paper including its various representations as a Nested Graph Condition (NGC), a Simple Graph Discrimination Network (SGDN) and a Simple Graph Discrimination Tree (SGDT), for which this paper showed equivalence in terms of their answer sets.

\section{B.1. Complex Query}

The type graph for the complex query is shown in Figure 6 (a) along with an example graph in (b). The query searches for pairs of Tags $t$ and Persons $s$ such that the Tag is new in the Posts by a friend $p$ of $s$.

(a)

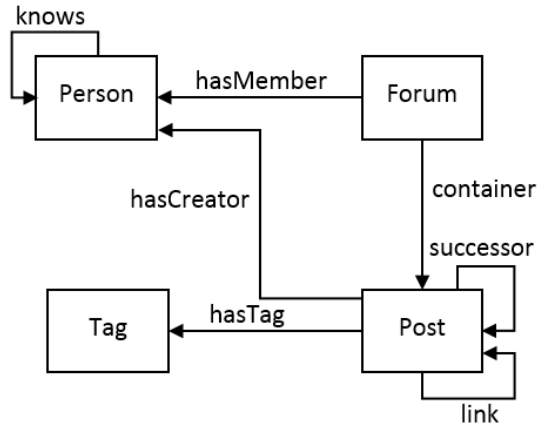

(b)

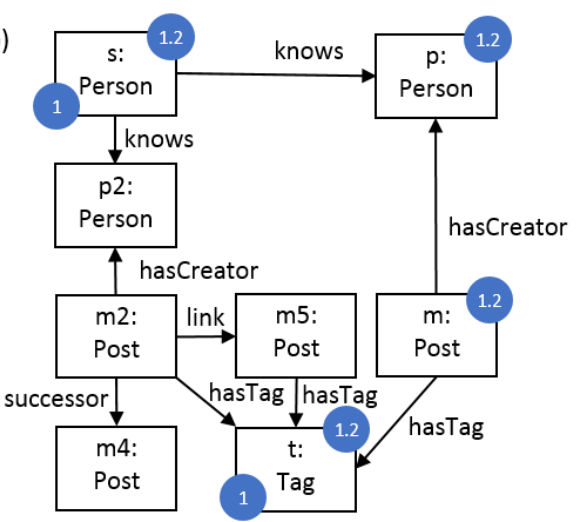

Figure 6: Excerpt of social network type graph and an example graph $G$

This query can be decomposed into the set of subconditions $L_{i}$ illustrated by the morphisms of Figure 7, which extends Figure 3 (a). Morphisms are identified by equally named nodes.

1. In order to be a Post of a friend, the considered Post $m$ must have been created (hasCreator) by a second Person that Person $s$ knows, and the Tag must be linked (hasTag) to the Post $\left(L_{1.2}\right)$.

a) However, the found Tag must not be inherited from a linked Post $(m 5)$ $\left(\neg \exists L_{1.2 .1}\right)$.

b) Furthermore, in order to be the latest Post, Post $m$ must have no successor Post $(m 3)\left(\neg \exists L_{1.2 .2}\right)$. 

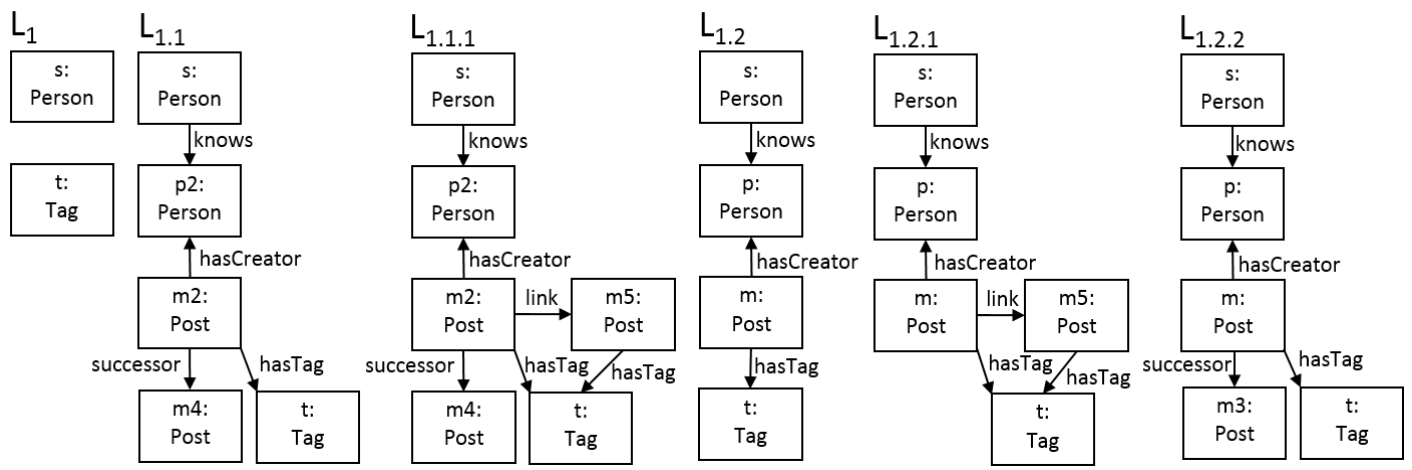

Figure 7: Complete set of graphs employed in the morphisms for the complex query and its subconditions

2. In order to be new, the Tag must not have been used by any former Post of any friend $\left(\neg \exists L_{1.1}\right)$.

a) However, similarly to $1 \mathrm{a}$ only Tags that are not simply inherited from a linked Post should be considered in this context $\left(\neg \exists L_{1.1 .1}\right)$.

Figure 6(b) shows an example graph where occurrences for the positive sentences $L_{1}$ and $L_{1.2}$ are depicted as markers in form of blue circles with the respective number. The blue markers (1) denote the only correct answer for the query. Thereby the required match for the positive subquery $L_{1.2}$ depicted by the markers (1.2) is such that indeed no match exists for the negative subconditions $L_{1.2 .1}$ and $L_{1.2 .2}$. Furthermore, as required no match for the negative subcondition $L_{1.1}$ consistent with $L_{1}$ exists such that no match for the negative subcondition $L_{1.1 .1}$ of $L_{1.1}$ can be found. Consequently, no match for $L_{1.1}$ is visualized.

\section{B.2. $\mathrm{NGC} c_{1}$}

The complex query as informally stated in the previous section translates into:

$$
\begin{aligned}
& \text { NGC } c_{1}=c_{1.1} \wedge c_{1.2} \\
& \text { with } \\
& c_{1.1}=\neg \exists\left(n_{1.1}: L_{1} \rightarrow L_{1.1}, c_{1.1 .1}\right), \\
& c_{1.2}=\exists\left(p_{1.2}: L_{1} \rightarrow L_{1.2}, c_{1.2 .1} \wedge c_{1.2 .2}\right), \\
& c_{1.1 .1}=\neg \exists\left(n_{1.1 .1}: L_{1.1} \rightarrow L_{1.1 .1}, \text { true }\right), \\
& c_{1.2 .1}=\neg \exists\left(n_{1.2 .1}: L_{1.2} \rightarrow L_{1.2 .1}, \text { true }\right), \text { and } \\
& c_{1.2 .2}=\neg \exists\left(n_{1.2 .2}: L_{1.2} \rightarrow L_{1.2 .2}, \text { true }\right)
\end{aligned}
$$


This is illustrated graphically in Figure 8 (a) where the above NGC $c_{1}$ takes an AST-like (Abstract Syntax Tree) form.

According to Proposition 1 of this paper, an equivalent Simple Graph Discrimination Tree (SGDT) can be constructed from $c_{1}$ as depicted in Figure 8 (b).

\section{B.3. Example SGDN}

The example query can also be expressed as a SGDN (Simple Graph Discrimination Network) as illustrated in Figure 9 (a), where the node $r_{1.1 .1 s}$ is reused as input by both $r_{1.1 s}$ and $r_{1.2 s}$ nodes.

For this GDN, simple Marking Rules (SMR) are defined to create markings for the found matches on the host graph when the query is executed. These marking rules are depicted in their compact notation in Figure 10

The simple marking rule

$r_{1 s}=\left(L_{1 s} \rightarrow R_{1 s},\left(\exists L_{1 s} \rightarrow P_{1}^{1}\right) \wedge\left(\nexists L_{1 s} \rightarrow N_{1}^{1}\right) \wedge\left(\nexists L_{1 s} \rightarrow N_{2}^{1}\right)\right)$

and its application condition

$a c_{L_{1 s}}=\left(\exists L_{1 s} \rightarrow P_{1}^{1}\right) \wedge\left(\nexists L_{1 s} \rightarrow N_{1}^{1}\right) \wedge\left(\nexists L_{1 s} \rightarrow N_{2}^{1}\right)$

is shown graphically in Figure 11 expressed in a less compact notation.

The application condition requires with the PAC $P_{1}^{1}$ that rule $r_{1.2 s}$ has created a marking for $L_{1.2 s}$ w.r.t. $L_{1 s}$ and by the NAC $N_{2}^{1}$ that conversely, rule $r_{1.1 s}$ did not create a marking for $L_{1.1 s}$ w.r.t. $L_{1 s}$. This is shown in the figure by dashed round circles with a crossed circle indicating a negative application as shown in the upper left corner of the figure. In addition, a marking $N_{1}^{1}$ must not already exist on the matched subgraph as also indicated by a cross in the upper left corner of Figure 11 .

Given that the application condition holds, marking rule $r_{1 s}$ can be applied to the host graph as illustrated by the LHS and RHS of the rule in the bottom part of Figure 11 .

\section{B.4. Example SGDT}

The SGDN of the previous section can be transformed into the equivalent SGDT of Figure 9 (b) according to Lemma 2. This is shown in Figure 9 (b) where the node $L_{1.1 .1 s}$ of (a) has been duplicated in (b) as indicated by the grayed box in order to constitute a tree.

The complete set of SMRs for this SGDT partially shown in Figure 5 (a) is presented in Figure 12. 
(a)

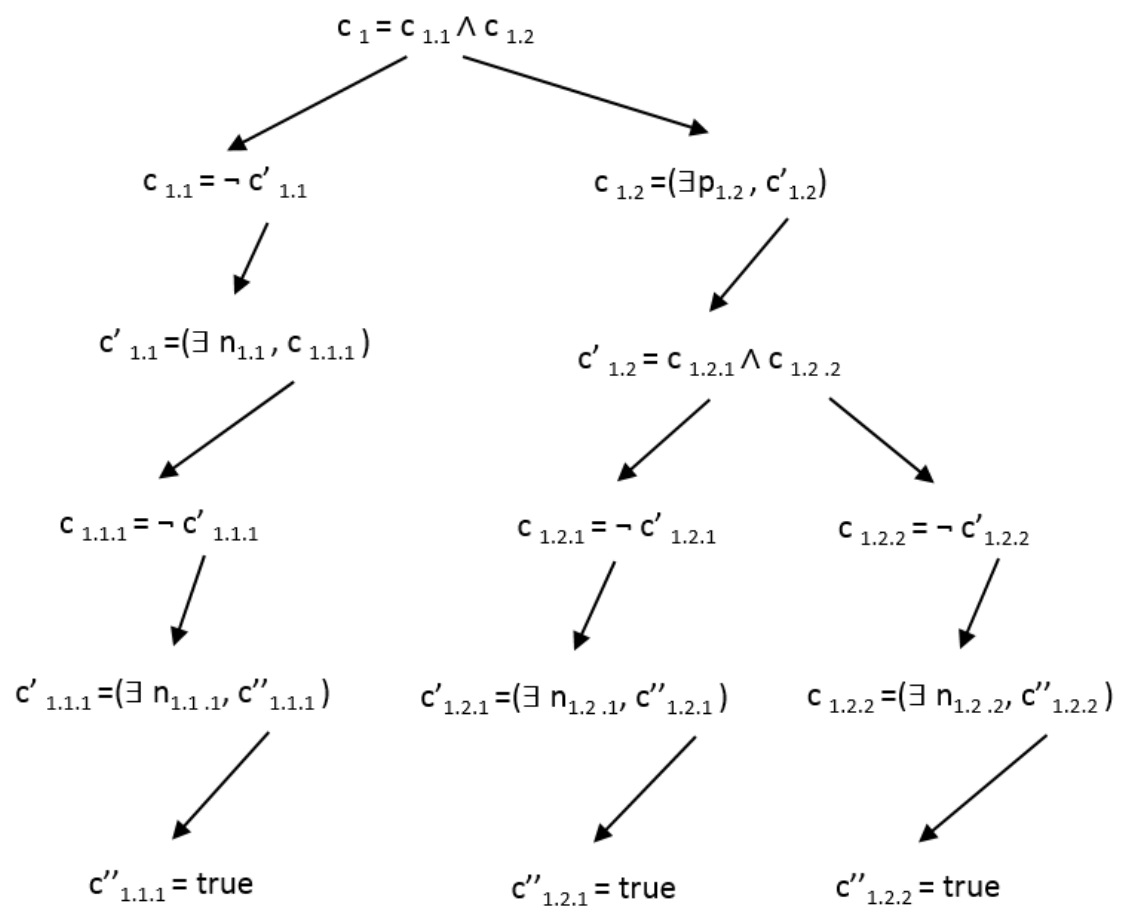

(b)

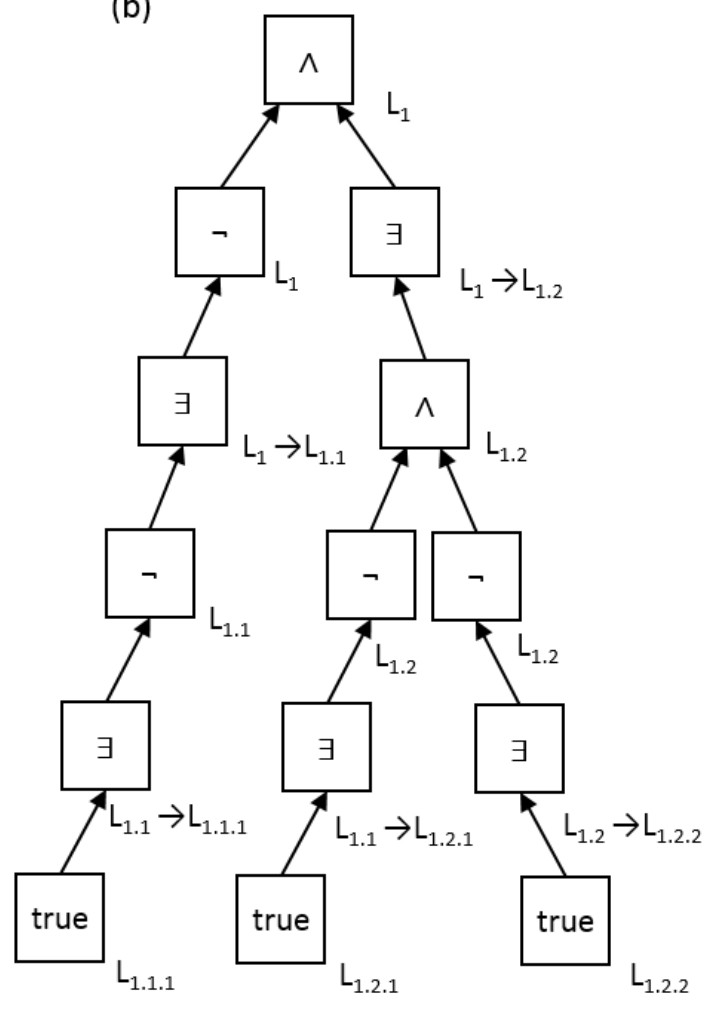

Figure 8: The NGC $c_{1}$ decomposed intofits subconditions and the related SGDT constructed according to Proposition 1 


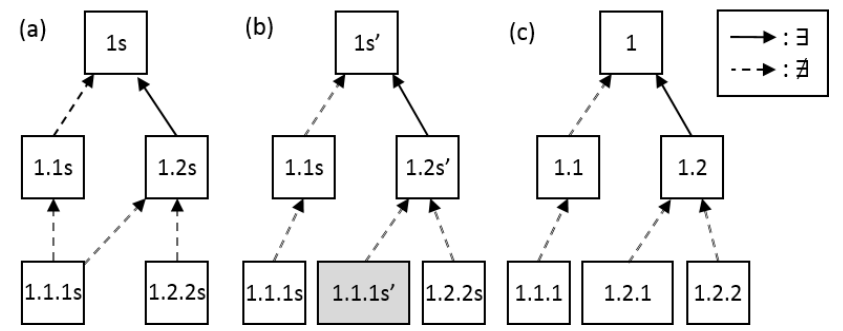

Figure 9: The SGDN decomposed into its subnodes and the related SGDT constructed according to Lemma 2
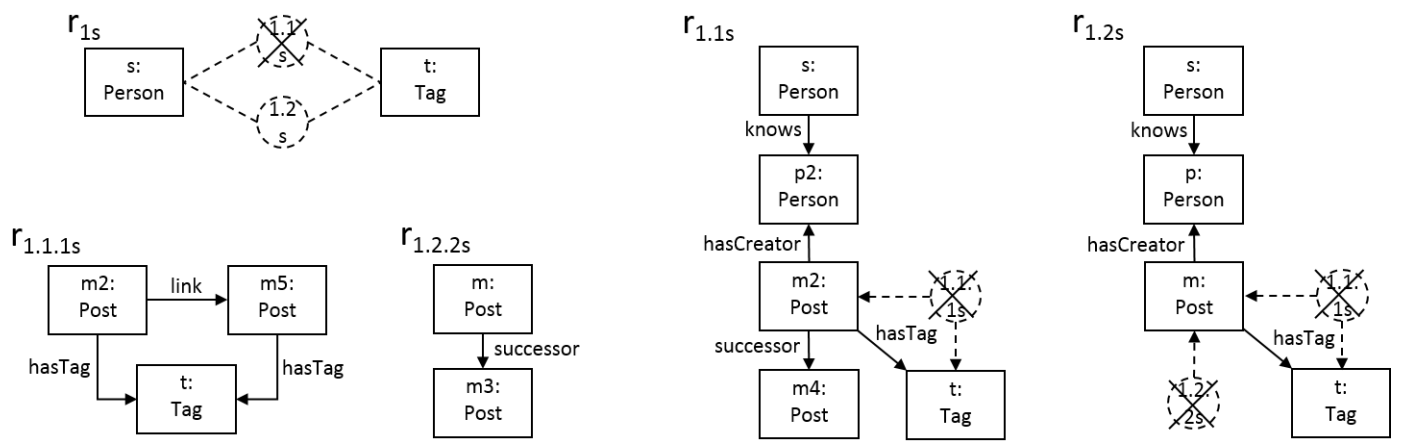

Figure 10: SMRs for the SGDN of the social network example

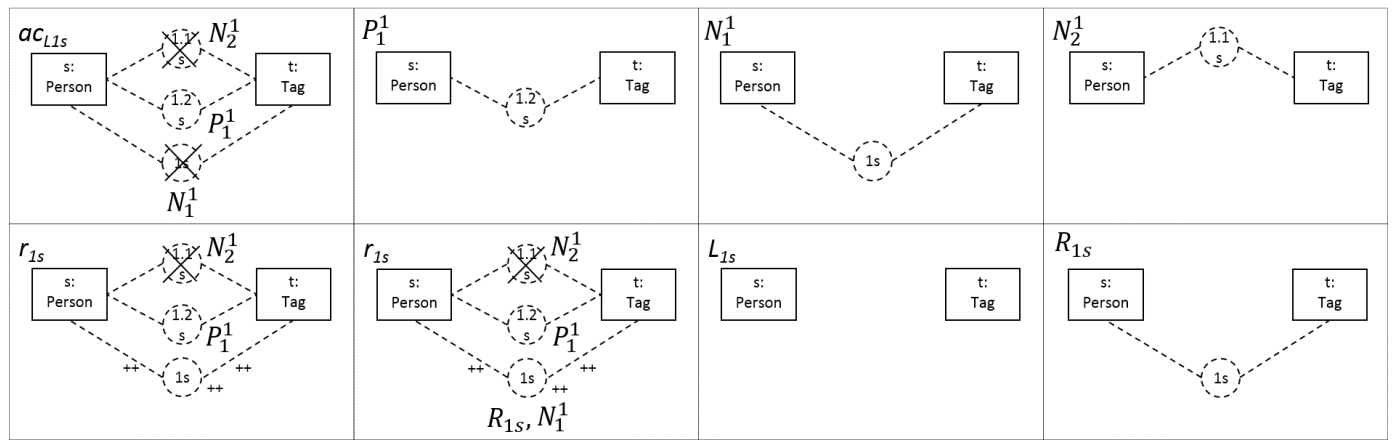

Figure 11: A diagram for the application condition $a c_{L_{1 s}}$ and simple marking rule $r_{1 s}$ of the example SGDN 

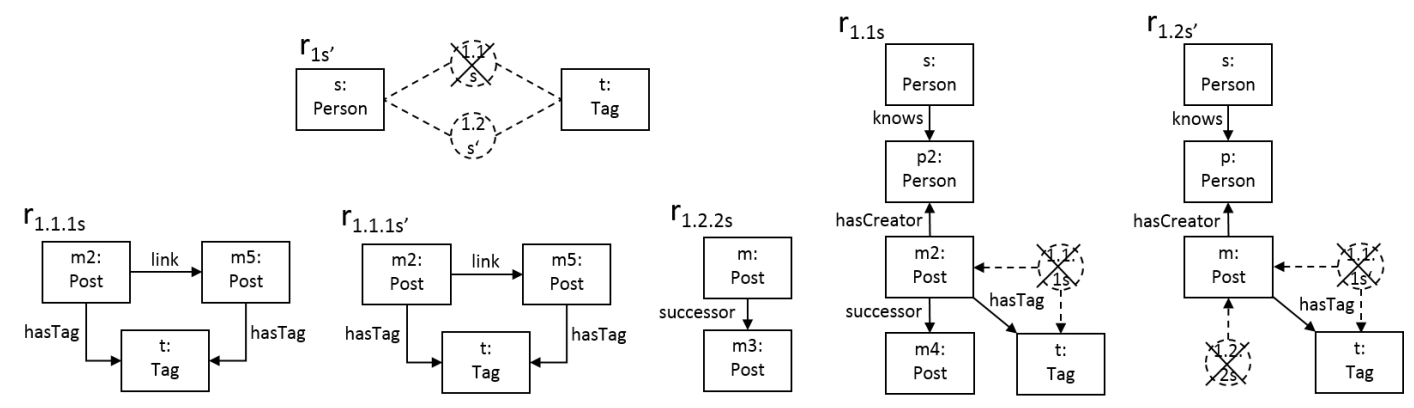

Figure 12: Complete set of SMRs for the example SGDT

\section{B.5. SGDT with Maximal Contexts}

In order to be as equally expressive as NGCs, SGDTs must be able to carry the same context provided for nested NGCs during their evaluation. For this, the SGDT needs to be extended such that SMRS also mark the elements from their parent rules (see Lemma 8 and Proposition 2). This is shown in Figure 13 where the complete set of SMRs, partially shown in Figure 5 (b) for the SGDT of Figure 9 (c) is shown. Maximal context marking links that have been added w. r. t. this maximal context generation process are displayed as orange dashed lines and corresponding available maximal context elements are identified as orange nodes and links.
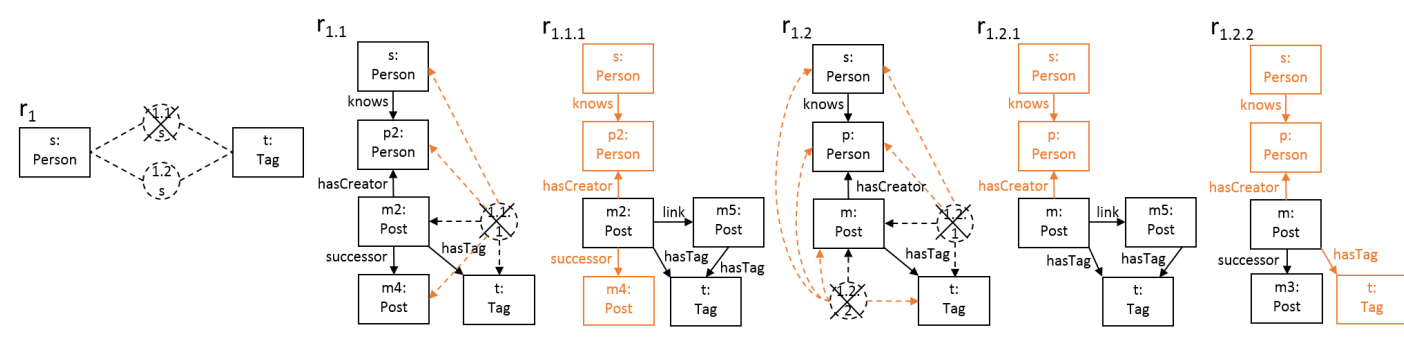

Figure 13: Complete set of SMRs for the example SGDT with maximal rule contexts

Note that for the two modified SMRs of the SGDT with maximal context, it holds that the presented rules $r_{1.1}$ and $r_{1.2}$ already have maximal context and reference directly related rules $r_{1.1 .1}, r_{1.2 .1}$, and $r_{1.2 .2}$. The maximal context of these latter rules is generated according to the construction of Lemma 7 , which includes 
an additional disjunction resp. conjunction for all alternative cases for context propagation. In particular for rule $r_{1.1 .1}$ two alternative contexts were generated, but one of them can be omitted again because of the cardinality constraint that there is no succesor edge as well as a link edge between a Post and another Post at the same time. This constraint expresses that it is not possible to link to a successor post that can not be created yet. For rule $r_{1.2 .1}$ and rule $r_{1.2 .2}$ no extra alternatives arise in the first place.

\section{B.6. Equivalence of the Example SGDT and $c_{1}$ According to Proposition 1}

As depicted in Figure 8 (a) we can represent the NGC $c_{1}$ in a form consistent with the one employed in Proposition 1 where only the operators $\neg, \wedge$, and $\exists$ and the constant true for given $L_{\ldots}$ are employed (see Figure 7 for the definition of the monomorphisms $n_{\ldots}$. resp $p_{\text {... }}$ ). For this form of NGC, we can then also construct the equivalent SGDT according to Proposition 1 as depicted in Figure 8 (b). 



\section{Aktuelle Technische Berichte des Hasso-Plattner-Instituts}

$\begin{array}{cll}\text { Band } & \text { ISBN } & \text { Titel } \\ 105 & 978-3-86956-360-2 & \begin{array}{l}\text { Proceedings of the Third HPI } \\ \text { Cloud Symposium } \\ \text { "Operating the Cloud" } 2015\end{array} \\ 104 & 978-3-86956-355-8 & \begin{array}{l}\text { Tracing Algorithmic Primitives in } \\ \text { RSqueak/VM }\end{array}\end{array}$

978-3-86956-345-9 Proceedings of the 9th Ph.D. retreat of the HPI Research School on service-oriented systems engineering

978-3-86956-339-8 Efficient and scalable graph view maintenance for deductive graph databases based on generalized discrimination networks

\section{8-3-86956-333-6}

978-3-86956-334-

978-3-86956-324-4

Inductive invariant checking with partial negative application conditions semantics and language testing with RML

Seminar on Event Processing Systems for Business Process Management Systems Interactive Content in a Live Environment Parts without a whole? : The current state of Design Thinking practice in organizations adaptive systems of systems : terms, characteristics, requirements and scenarios

978-3-86956-320-6 Proceedings of the 8th Ph.D.

\section{Autoren / Redaktion}

Estee van der Walt, Jan Lindemann, Max Plauth, David Bartok (Hrsg.)

Lars Wassermann, Tim Felgentreff, Tobias Pape, Carl Friedrich Bolz, Robert Hirschfeld

Tim Felgentreff, Robert Hirschfeld, Todd Millstein, Alan Borning

Anne Baumgraß, Andreas Meyer, Mathias Weske (Hrsg.)

Philipp Otto, Jaqueline Pollak, Daniel Werner, Felix Wolff, Bastian Steinert, Lauritz Thamsen, Macel Taeumel, Jens Lincke, Robert Krahn, Daniel H. H. Ingalls, Robert Hirschfeld

Christoph Meinel, Hasso Plattner, Jürgen Döllner, Mathias Weske, Andreas Polze, Robert Hirschfeld, Felix Naumann, Holger Giese, Patrick Baudisch, Tobias Friedrich (Hrsg.)

Thomas Beyhl, Holger Giese

Johannes Dyck, Holger Giese

Jan Schmiedgen, Holger Rhinow, Eva Köppen, Christoph Meinel

Sebastian Wätzoldt, Holger Giese retreat of the HPI research school on service-oriented systems engineering

Christoph Meinel, Hasso Plattner, Jürgen Döllner, Mathias Weske, Andreas Polze, Robert Hirschfeld, Felix Naumann, Holger Giese, Patrick Baudisch 


ISBN 978-3-86956-372-5

ISSN 1613-5652 